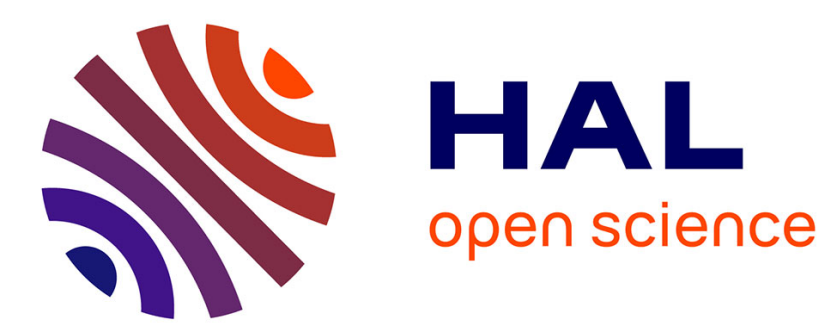

\title{
On the normality of the null-fiber of the moment map for theta- and tori representations
}

\author{
Michael Bulois
}

\section{To cite this version:}

Michael Bulois. On the normality of the null-fiber of the moment map for theta- and tori representations. Journal of Algebra, 2018, 507, pp.502-524. 10.1016/j.jalgebra.2018.04.011 . hal-01558530

\author{
HAL Id: hal-01558530 \\ https://hal.science/hal-01558530
}

Submitted on 11 Jul 2017

HAL is a multi-disciplinary open access archive for the deposit and dissemination of scientific research documents, whether they are published or not. The documents may come from teaching and research institutions in France or abroad, or from public or private research centers.
L'archive ouverte pluridisciplinaire HAL, est destinée au dépôt et à la diffusion de documents scientifiques de niveau recherche, publiés ou non, émanant des établissements d'enseignement et de recherche français ou étrangers, des laboratoires publics ou privés. 


\title{
ON THE NORMALITY OF THE NULL-FIBER OF THE MOMENT MAP FOR $\theta$ - AND TORI REPRESENTATIONS
}

\author{
MICHAEL BULOIS
}

\begin{abstract}
Let $(G, V)$ be a representation with either $G$ a torus or $(G, V)$ a locally free stable $\theta$-representation. We study the fiber at 0 of the associated moment map, which is a commuting variety in the latter case. We characterize the cases where this fiber is normal. The quotient (i.e. the symplectic reduction) turns out to be a specific orbifold when the representation is polar. In the torus case, this confirms a conjecture stated by C. Lehn, M. Lehn, R. Terpereau and the author in a former article. In the $\theta$-case, the conjecture was already known but this approach yield another proof.
\end{abstract}

MSC 2010: 14L24, 17B20, 17B70, 20G05 , 53D20.

Keywords: theta-representations, torus, commuting variety, moment map, symplectic reduction, orbifold

\section{Contents}

Introduction $\quad 1$

1. Generalities 3

2. Null-fiber of the moment map - torus case 6

3. Symplectic reduction for tori representations 8

4. Null-fiber of the moment map - $\theta$ case 13

References $\quad 20$

\section{INTRODUCTION}

Let $\mathbb{k}$ be an algebraically closed field of characteristic zero. Let $(G, V)$ be a representation of a connected reductive algebraic group $G$ on a finite dimensional $\mathbb{k}$-vector space $V$. Let $\mathfrak{g}$ be the Lie algebra of $G$. We define the moment map

$$
\mu:\left\{\begin{array}{ccc}
V \oplus V^{*} & \rightarrow & \mathfrak{g}^{*} \\
(x, \varphi) & \mapsto & (g \mapsto \varphi(g \cdot x))
\end{array}\right.
$$

Among the fibers of the moment map, the most special one is $\mu^{-1}(0)$. When the representation $(G, V)$ is the adjoint action of $G$ on $\mathfrak{g}, \mu^{-1}(0)$ can be identified wih the commuting scheme of $\mathfrak{g}$. According to a long-standing conjecture, this scheme should be normal. One of the aim of this work is to study the normality of the scheme $\mu^{-1}(0)$ in some nice cases. For instance, if $(G, V)$ is visible and locally free, we already know that $\mu^{-1}(0)$ is a complete intersection and the normality question reduces to singular locus questions thanks to Serre's criterion (see e.g. Corollary 1.7 and Remark 1.8). Using this, Panyushev [Pan] has shown that $\mu^{-1}(0)$ is reduced 
and normal in the case of the representation associated to a symmetric Lie algebra of maximal rank.

There are some natural generalizations of adjoint representations and symmetric Lie algebras: the so-called $\theta$-representations ( or $\theta$-groups) of Vinberg [Vin] and, more generally, the polar representations of Dadok and Kac [DK]. Recall that a $\theta$-representation is a representation $(G, V)$ isomorphic to some $\left(H_{0}, \mathfrak{h}_{1}\right)$ where $H$ is a connected reductive group acting on its Lie algebra $\mathfrak{h}$, with $\mathfrak{h}$ equipped with a $\mathbb{Z}_{m}$-grading $\bigoplus_{i \in \mathbb{Z}_{m}} \mathfrak{h}_{i}$ and where $H_{0}$ is the connected subgroup of $H$ whose Lie algebra is $\mathfrak{h}_{0}$. It turns out that the geometry of $\mu^{-1}(0)$ can be a bit more involved in this setting of $\theta$-representations than for the classical adjoint representation. For instance, it is possible to find examples of such representations where $\mu^{-1}(0)$ is non-irreducible [Pan, Bu11], non-reduced or irreducible non-normal [BLLT]. In this paper, we classify the locally free stable $\theta$-representations for which $\mu^{-1}(0)$ is normal. It turns out (Theorem 4.6) that this normality property holds in all the classical cases and we find only 5 non-normal examples of exceptional cases, each of rank 1 . We take the opportunity to generalize some properties of decompositions classes to the $\theta$-cases in Section 4 . The $\theta$-case is partly based on the study of some specific tori representations. This is one motivation for studying $\mu^{-1}(0)$ for a general representation of a torus in Section 2. The fiber turns out to be normal as soon as it is irreducible and we give a combinatorial criterion for this in terms of the weights of the representation (Theorem 2.2).

The variety $\mu^{-1}(0)$ is also involved in the symplectic reduction $\left(V \oplus V^{*}\right) / / / G:=$ $\mu^{-1}(0) / / G$ where $X / / G$ denotes the categorical quotient of an affine $G$-variety $X$. If $\mu^{-1}(0)$ is normal, then the symplectic reduction is also normal. Following a theorem of Joseph [Jos] in the Lie algebra case, and a conjecture stated in [BLLT] in the complex case (see conjecture 1.3 here), the variety $\left(V \oplus V^{*}\right) / / / G$ should be isomorphic to a specific orbifold whenever $(G, V)$ is visible and polar. The results of the present paper yield a proof of this conjecture when $G$ is a torus (Corollary $3.10)$, and allows to recover it when $(G, V)$ is a stable locally free $\theta$-representation (Remark 4.13). In the torus case, we also get that that the visibility assumption is necessary (Proposition 3.9) but that the scheme $\left(V \oplus V^{*}\right) / / G$ is nevertheless reduced (Theorem 2.2) and normal (Proposition 3.4) in general.

One should note that, as the torus case suggests, we put light on representations which are far from being irreducible. Part of the results concerning tori (e.g., the normality of the symplectic reduction) were written independantly in the recent preprint [HSS]. The general philosophy of [HSS] is to focus on "large" representations (ex: 2-large) whereas the polar representations are "small".

Acknowledgements. Section 4 of this paper was essentially included in early versions of [BLLT]. I want to thank my co-authors to allow me to reuse this material here.

This work has benefited from the support of the project GeoLie (ANR-15-CE400012) and of the LABEX MILYON (ANR-10-LABX-0070) of Université de Lyon 
within the program "Investissements d'Avenir" (ANR-11-IDEX-0007) operated by the French National Research Agency (ANR).

\section{Generalities}

In this section, we introduce some classical material to study null fibers of moment maps. Even if some results are stated in a greater generalities, most of this section is heavily inspired by [Pan]. Popov's article [Po] is also a source of inspiration.

We work over an algebraically closed field $\mathbb{k}$ of characteristic zero. We use the language of scheme even if these schemes will always be separated of finite type over $\mathbb{k}$ and will often be reduced, i.e. varieties. We do not require that the varieties are irreducible. When speaking about the irreducible components of a scheme, we always mean the irreducible components of the corresponding reduced variety and we do not consider embedded components.

If $A$ is a subset of a vector space, $\langle A\rangle$ stands for the subspace generated by $A$.

Definition 1.1. An element $x \in V$ is said to be

- semisimple if G.x is closed,

- nilpotent if $0 \in \overline{G . x}$.

Equivalently, $x$ is nilpotent if and only if $\pi(x)=\pi(0)$ where $\pi: V \rightarrow V / / G$ is the categorical quotient map.

Definition 1.2. The representation $(G, V)$, is said to be

- locally free, if $\operatorname{dim} G . x=\operatorname{dim} G$ for some $x \in V$.

- visible, if there are finitely many nilpotent $G$-orbits.

- stable, if there is an open subset of $V$, consisting of semisimple elements

- polar, if there exists $v \in V$ such that $\operatorname{dim} \mathfrak{c}_{v}=\operatorname{dim} V / / G$ where $\mathfrak{c}_{v}:=$ $\{x \in V \mid \mathfrak{g} \cdot x \subset \mathfrak{g} \cdot v\}$. We then say that $\mathfrak{c}_{v}$ is a Cartan subspace of $V$.

Examples of visible and stable representations are given by the adjoint action of $G$ on $\mathfrak{g}$, or a $\theta$-representation arising from a $\mathbb{Z}_{2}$-grading (so-called symmetric Lie algebras). In general, $\theta$-representations are always visible but not necessarily stable [Vin]. Polar representations, as introduced in [DK], can be neither stable nor visible. Examples of locally free representations are given by symmetric Lie algebras of maximal rank. It is worth noting that no adjoint representation $(G, \mathfrak{g})$ is locally free.

When $k=\mathbb{C}$ and $(G, V)$ is polar, we write $\mathfrak{c}$ and $\mathfrak{c}^{\vee}$ for dual Cartan subspaces of $V$ and $V^{*}$, following [BLLT, (3.4), (3.6)], together with dual decompositions $V=\mathfrak{c} \oplus(\mathfrak{g} \cdot \mathfrak{c}) \oplus U$ and $V^{*}=\mathfrak{c}^{\vee} \oplus\left(\mathfrak{g} \cdot \mathfrak{c}^{\vee}\right) \oplus U^{\vee}$. Write $W$ for the Weyl group $N_{G}(\mathfrak{c}) / C_{G}(\mathfrak{c})$. The following was Conjecture $A$ in [BLLT].

Conjecture 1.3. Assume that $(G, V)$ is visible and polar, then we have an isomorphism of Poisson varieties $\left(V \oplus V^{*}\right) / / / G \cong\left(\mathfrak{c} \oplus \mathfrak{c}^{\vee}\right) / W$.

Remark 1.4. Assume $\mathbb{k}=\mathbb{C}$. From [BLLT, Proposition 3.3\&3.5], we have a injective natural morphism $\mathfrak{c} \times \mathfrak{c}^{\vee} / W \rightarrow \mu^{-1}(0) / / G$ of Poisson varieties. Since $\mathfrak{c} \oplus \mathfrak{c}^{\vee} / W$ is normal, Conjecture 1.3 is equivalent to: 
(1) The morphism $\mathfrak{c} \times \mathfrak{c}^{\vee} / W \rightarrow \mu^{-1}(0) / / G$ is dominant (i.e. it is bijective.)

(2) $\mu^{-1}(0) / / G$ is normal

When studying the null fiber of the moment map, it is enlightening to study the modality of $(G, V)$. The modality of a $G$-subvariety $X \subseteq V$ is defined as

$$
\bmod (G, X):=\max _{Y \subseteq X}\left(\min _{x \in Y}\left(\operatorname{codim}_{Y} G \cdot x\right)\right)
$$

where the maximum is taken over all irreducible subvarieties $Y \subseteq X$.

In the remaining of the section we will consider a finite cover of $V, V=\bigcup_{i} J_{i}$ such that

- Each $J_{i}$ is $G$-stable, irreducible and locally closed in $V$ of constant orbit dimension (that is $\exists m_{i} \in \mathbb{N}, \forall x \in J_{i}, \operatorname{dim} G \cdot x=m_{i}$ ),

- $\overline{J_{i}}=\overline{J_{j}} \Rightarrow i=j$.

Exemples of such cover includes sheets of $(G, V)$ or decomposition classes in the case of the adjoint action. Note that if the cover is disjoint $V=\bigsqcup_{i} J_{i}$, then the second condition follows from the first one. Since the $J_{i}$ are of constant orbit dimension, we have $\bmod \left(G, J_{i}\right)=\operatorname{dim} J_{i}-m_{i}$. Moreover, from finiteness and locally closeness, each irreducible subvariety $Y \subset V$ intersects at least one $J_{i}$ as a dense open subset of $Y$ and we have $\min _{x \in Y} \operatorname{codim}_{Y} G \cdot x=\bmod \left(G, J_{i}\right)$. Hence

$$
\bmod (G, V)=\max _{i} \bmod \left(G, J_{i}\right)
$$

Given a subvariety $X \subseteq V$, we write

$$
\left(\mu^{-1}(0)\right)_{X}:=\left\{(x, \varphi) \in \mu^{-1}(0) \mid x \in X\right\}=\left\{(x, \varphi) \in X \times V^{*} \mid \varphi \in(\mathfrak{g} \cdot x)^{\perp}\right\}
$$

seen as a subvariety of $\mu^{-1}(0)$. This allows for instance to decompose $\mu^{-1}(0)$, as a finite union:

$$
\mu^{-1}(0)=\bigcup_{i}\left(\mu^{-1}(0)\right)_{J_{i}}
$$

Lemma 1.5. (i) Each $\left(\mu^{-1}(0)\right)_{J_{i}}$ is an irreducible and locally closed subvariety of $\mu^{-1}(0)$ of dimension $\operatorname{dim} V+\bmod \left(G, J_{i}\right)$.

(ii) Each irreducible component of $\mu^{-1}(0)$ is of the form $\overline{\left(\mu^{-1}(0)\right)_{J_{i}}}$ for some $i$.

(iii) There is a bijection between the set of irreducible components of $\mu^{-1}(0)$ of maximal dimension $\operatorname{dim} V+\bmod (G, V)$ and the set of $J_{i}$ with maximal modality $\bmod \left(G, J_{i}\right)=\bmod (G, V)$.

Proof. (i) From (2), $\left(\mu^{-1}(0)\right)_{J_{i}}$ is a subbundle in $J_{i} \times V^{*}$ of codimension $m_{i}$. In particular, it is an irreducible subvariety of $\mu^{-1}(0)$ of dimension

$$
\operatorname{dim} V+\operatorname{dim} J_{i}-m_{i}=\operatorname{dim} V+\bmod \left(G, J_{i}\right)
$$

(ii) is clear from (i) and (3).

(iii) By considering projection on the first variable, $\overline{\left(\mu^{-1}(0)\right)_{J_{i}}}=\overline{\left(\mu^{-1}(0)\right)_{J_{j}}}$ can happen only if $\overline{J_{i}}=\overline{J_{j}}$, that is only if $i=j$. The bijection is then clear from (i) and (ii). 
Another classical result is the following characterization of the smooth locus of $\mu^{-1}(0)$. If $y$ belongs to a $G$-module $Y$ (e.g. $Y=V$ or $Y=V \oplus V^{*}$ ), we let $\mathfrak{g}^{y}:=\{g \in \mathfrak{g} \mid g \cdot y=0\}$ be the centralizer of $y$ in $\mathfrak{g}$.

Proposition 1.6. The following assertions are equivalent for an element $(x, \varphi) \in$ $\mu^{-1}(0) \subset V \oplus V^{*}$

(i) $(x, \varphi)$ is a smooth point of the scheme $\mu^{-1}(0)$

(ii) $(x, \varphi)$ belongs to a unique irreducible component $\overline{\left(\mu^{-1}(0)\right)_{J_{i}}}$ for some $J_{i}$, and $\operatorname{dim} \mathfrak{g}^{x, \varphi}=\bmod \left(G, J_{i}\right)+\operatorname{dim} \mathfrak{g}-\operatorname{dim} V$

Proof. The tangent space of $\mu^{-1}(0)$ at $z=(x, \varphi)$ is $\operatorname{Ker} d_{z} \mu$. The dual map of $d_{z} \mu$ has the following expression



In particular, $\operatorname{Ker}\left(d_{z}(\mu)\right)^{*}=\{g \in \mathfrak{g} \mid g \cdot x=0=g \cdot \varphi\}=\mathfrak{g}^{x, \varphi}$. Hence $\operatorname{dim} \operatorname{Ker} d_{z} \mu=$ $2 \operatorname{dim} V-\operatorname{dim} \mathfrak{g}+\operatorname{dim} \mathfrak{g}^{x, \varphi}$.

Recall that smooth points on a scheme belong to a unique irreducible component. Let $(x, \varphi)$ belong to a unique irreducible component $\overline{\left(\mu^{-1}(0)\right)_{J_{i}}}$ for some $J_{i}$. Since $\operatorname{dim}\left(\mu^{-1}(0)\right)_{J_{i}}=\operatorname{dim} V+\bmod \left(G, J_{i}\right)$ (Lemma 1.5), we get that $(x, \varphi)$ is a smooth point of $\mu^{-1}(0)$ if and only if $\operatorname{dim} V-\operatorname{dim} \mathfrak{g}+\operatorname{dim} \mathfrak{g}^{x, \varphi}=\bmod \left(G, J_{i}\right)$, hence the equivalence.

Corollary 1.7. Assume that $\bmod (G, V)=\operatorname{dim} V-\operatorname{dim} G$. Then

(i) $(G, V)$ is locally free.

(ii) $\mu^{-1}(0)$ is a complete intersection of dimension $2 \operatorname{dim} V-\operatorname{dim} G$.

(iii) The smooth points of the scheme $\mu^{-1}(0)$ are the points $(x, \varphi)$ satisfying $\mathfrak{g}^{x, \varphi}=\{0\}$.

(iv) $\mu^{-1}(0)$ is irreducible if and only if $\bmod \left(G, J_{i}\right)<\bmod (G, V)$, for any $i$ such that $\overline{J_{i}} \neq V$. If it is the case, the scheme $\mu^{-1}(0)$ is also reduced

(v) $\mu^{-1}(0)$ is reduced and normal if and only if it is irreducible and the following holds

(5) $\forall i,\left[\bmod \left(G, J_{i}\right)=\bmod (G, V)-1 \Rightarrow\left(\exists(x, \varphi) \in\left(\mu^{-1}(0)\right)_{J_{i}}\right.\right.$ s.t. $\left.\left.\mathfrak{g}^{x, \varphi}=\{0\}\right)\right]$

Proof. (i) Let $i_{0}$ be the index such that $\overline{J_{i_{0}}}=V$. Then $m_{i_{0}}$ is the maximum orbit dimension in $V$ and we get $\bmod (G, V) \geqslant \bmod \left(G, J_{i_{0}}\right)=\operatorname{dim} V-m_{i_{0}} \geqslant \operatorname{dim} V-\operatorname{dim} G$. From the hypothesis, we have equalities so $m_{i_{0}}=\operatorname{dim} G$.

(ii) The irreducible components of maximal dimension of $\mu^{-1}(0)$ have codimension $2 \operatorname{dim} V-(\operatorname{dim} V+\bmod (G, V))=\operatorname{dim} G$ in $V \oplus V^{*}$. Since $\mu^{-1}(0)$ is defined in $V \oplus V^{*}$ by $\operatorname{dim} \mathfrak{g}$ equation, (ii) follows.

(iii) From (ii), $\mu^{-1}(0)$ is equidimensional and its irreducible components have dimension $2 \operatorname{dim} V-\operatorname{dim} G$. From proof of Proposition 1.6, we see that the tangent space of $\mu^{-1}(0)$ at $(x, \varphi)$ has dimension $2 \operatorname{dim} V-\operatorname{dim} \mathfrak{g}+\operatorname{dim} \mathfrak{g}^{x, \varphi}$. The result follows.

(iv) We have already seen in (i) that $\bmod \left(G, J_{i_{0}}\right)=\bmod (G, V)$ for $i_{0}$ such that $\overline{J_{i_{0}}}=V$. The characterization of the irreducibility of $\mu^{-1}(0)$ then follows from 
equidimensionality and Lemma 1.5 (iii). For $x \in J_{i_{0}}$, we have $\mathfrak{g}^{x}=\{0\}$. So $\left(\mu^{-1}(0)\right)_{J_{i_{0}}}$ is in the smooth locus of $\mu^{-1}(0)$ by (iii). Hence $\mu^{-1}(0)$ is reduced as soon as it is irreducible.

(v) Recall now that a variety is normal if and only if it satisfies Serre's condition $\left(S_{2}\right)$ and its singular locus has codimension at least 2 . The former is provided by (ii). Under the irreducibility assumption, the latter is equivalent to (5). Indeed, the singular locus of $\mu^{-1}(0)$ is a closed subset of $\bigcup_{i \neq i_{0}}\left(\mu^{-1}(0)\right)_{J_{i}}$. But, it follows from Lemma 1.5(i) that an irreducible component of a closed subset of $\bigcup_{i \neq i_{0}}\left(\mu^{-1}(0)\right)_{J_{i}}$ of codimension 1 in $\mu^{-1}(0)$ contains at least one $\left(\mu^{-1}(0)\right)_{J_{i}}$ with $\bmod \left(G, J_{i}\right)=\bmod (G, V)-1$.

Remark 1.8. In the notation of the proof of (i), we have, for a general representation $(G, V): \bmod (G, V) \geqslant \operatorname{dim} V-m_{i_{0}}$. The limit case $\bmod (G, V)=\operatorname{dim} V-m_{i_{0}}$ corresponds to condition $\left(\mathcal{F}_{0}\right)$ of [Pan], see $(2.2)$ and Corollary 2.5 in loc. cit. Then Proposition 3.1 in loc. cit. asserts that this condition hold when $(G, V)$ is visible. In particular, hypothesis of the above corollary are satisfied when $(G, V)$ is locally free and visible.

Similarily, condition $\left(\mathcal{F}_{1}\right)$ in loc. cit. is equivalent to the condition appearing in Corollary 1.7 (iv). Related statements to corollary 1.7 can be found in Theorems 2.4, 3.1 and 3.2 in loc. cit.

\section{Null-fiber of the MOMEnt MAP - TORUS CASE}

In this section $V$ is an $n$-dimensional representation of an $r$-dimensional torus $G=T \cong\left(\mathbb{k}^{\times}\right)^{r}$. The weight space $X^{*}(T)$ is isomorphic to $\mathbb{Z}^{r}$. We let $W_{\mathbb{Q}}:=$ $X^{*}(T) \otimes_{\mathbb{Z}} \mathbb{Q}$. It is a $\mathbb{Q}$-vector space of dimension $r$. Recall that the irreducible representations of $T$ are one-dimensional. So we can decompose $V=\bigoplus_{i=1}^{n} V_{i}$, with $T$ acting on $V_{i} \cong \mathbb{k}$ with weight $s_{i}=\left(s_{i}^{j}\right)_{j \in \llbracket 1, r \rrbracket} \in X^{*}(T)$. That is, the action of an element $t=\left(t_{j}\right)_{j \in \llbracket 1, r \rrbracket} \in T$ on an element $v=\left(v_{i}\right)_{i \in \llbracket 1, n \rrbracket} \in V$ is given by

$$
t \cdot v=\left(\left(\prod_{j=1}^{r} t_{j}^{\left(s_{i}^{j}\right)}\right) v_{i}\right)_{i} .
$$

We introduce the $n \times r$ matrix $S:=\left(s_{i}^{j}\right)_{i, j}$. Given $I \subset \llbracket 1, n \rrbracket$ or $i \in \llbracket 1, n \rrbracket$, we also define the $\# I \times r$-matrix $S_{I}:=\left(s_{i}^{j}\right)_{(i, j) \in I \times \llbracket 1, r \rrbracket}$ and the $(n-1) \times r$-matrix $S_{\hat{\imath}}:=S_{\llbracket 1, n \rrbracket \backslash\{i\}}$.

In the sequel we need a partition of $V$ by suitable strata. These are indexed by subsets $I \subset \llbracket 1, n \rrbracket$ and are given by

$$
J_{I}:=\left\{\left(v_{i}\right)_{i} \in V \mid v_{i} \neq 0 \Leftrightarrow i \in I\right\} .
$$

Remark 2.1. Each $J_{I}$ is a locally closed irreducible $T$-stable subvariety whose closure is $V_{I}:=\left\{\left(v_{i}\right)_{i} \in V \mid \forall i \notin I, v_{i}=0\right\}$.

Moreover, there is a $T$-equivariant action of $\left(\mathbb{C}^{\times}\right)^{n}$ on $V$ given by $\left(\lambda_{i}\right)_{i} \cdot\left(v_{i}\right)_{i}=$ $\left(\lambda_{i} v_{i}\right)_{i}$. The orbits of this action are precisely the strata $J_{I}$. Hence many geometric properties of the action of $T$ on a point $v \in V$ (dimension of orbit, nilpotency, semisimplicity, ...) are preserved along the stratum $J_{I}$ containing $v$. It will then 
be convenient to make the computations only at $v_{I}:=\left(\delta_{i \in I}\right)_{i}$ where $\delta$ is the Kronecker symbol.

It is now clear that $V=\bigsqcup_{I} J_{I}$ is a cover satisfying the assumptions of Section 1.

For $v \in V$, writing $X_{v}:=\left(\begin{array}{lll}v_{1} & & \\ & \ddots & \\ & & v_{n}\end{array}\right)$, we have

$$
\mathfrak{t} \cdot v=\left\langle\left(s_{i}^{j} v_{i}\right)_{i \in \llbracket 1, n \rrbracket} \mid j \in \llbracket 1, r \rrbracket\right\rangle=\operatorname{Im}\left(X_{v} S\right)=X_{v} \operatorname{Im}(S) .
$$

where $\operatorname{Im}(S)$ (resp. $\operatorname{Im}\left(X_{v} S\right)$ ) denote the image space of the linear maps $\mathbb{k}^{r} \rightarrow \mathbb{k}^{n}$ associated to $S$ (resp. $X_{v} S$ ) in the canonical basis. It follows from (6) that, for $v \in J_{I}$, we have

$$
\operatorname{dim} T \cdot v=\operatorname{rk} S_{I} .
$$

In particular, the modality of $J_{I}$ is given by

$$
\bmod \left(T, J_{I}\right)=\# I-\mathrm{rk} S_{I}=\operatorname{dim} \operatorname{Ker}\left({ }^{t} S_{I}\right) .
$$

where $\operatorname{Ker}\left({ }^{t} S_{I}\right)$ is the kernel of the linear map $\mathbb{k}^{\# I} \rightarrow \mathbb{k}^{r}$ associated to ${ }^{t} S_{I}$. Via the natural identifications $\mathbb{k}^{\# I} \cong V_{I} \subset V \cong \mathbb{k}^{n}, \operatorname{Ker}\left({ }^{t} S_{I}\right)$ is identified with $\operatorname{Ker}\left({ }^{t} S\right) \cap V_{I}$. Assume that $I_{1} \subset I_{2}$ are two subsets of $\llbracket 1, n \rrbracket$, then $\operatorname{Ker}\left({ }^{t} S\right) \cap V_{I_{1}} \subset \operatorname{Ker}\left({ }^{t} S\right) \cap V_{I_{2}}$. So

$$
I_{1} \subset I_{2} \Rightarrow \bmod \left(T, J_{I_{1}}\right) \leqslant \bmod \left(T, J_{I_{2}}\right)
$$

From (1), we get

$$
\bmod (T, V)=\bmod \left(T, J_{\llbracket 1, n \rrbracket}\right)=\operatorname{dim} V-\operatorname{rk} S .
$$

We can apply (2) to $X=J_{I}$ to define $\left(\mu^{-1}(0)\right)_{J_{I}}$. This yields a partition of $\mu^{-1}(0)$ into a disjoint union of irreducible locally closed (Lemma 1.5) subsets

$$
\mu^{-1}(0)=\bigsqcup_{I}\left(\mu^{-1}(0)\right)_{J_{I}}
$$

Theorem 2.2. Assume that $(T, V)$ is a reprentation with $T$ a torus.

Then, under above notation:

(i) $\mu^{-1}(0)$ is a reduced complete intersection of dimension $2 \operatorname{dim} V-\operatorname{rk} S$.

(ii) The irreducible components of $\mu^{-1}(0)$ are the subsets of the form $\overline{\left(\mu^{-1}(0)\right)_{J_{I}}}$ with $\operatorname{rk} S-\operatorname{rk} S_{I}=n-\# I$.

(iii) $\mu^{-1}(0)$ is irreducible if and only if, for any $i \in \llbracket 1, n \rrbracket$, we have $\operatorname{rk}\left(S_{\hat{\imath}}\right)=\operatorname{rk} S$.

(iv) $\mu^{-1}(0)$ is normal if and only if it is irreducible.

Proof. By Lemma 1.5, the irreducible components of $\mu^{-1}(0)$ of maximal dimension $\operatorname{dim} V+\bmod (T, V)=2 \operatorname{dim} V-\operatorname{rk} S(10)$ are the $\overline{\left(\mu^{-1}(0)\right)_{J_{I}}}$ with $\# I-\operatorname{rk} S_{I}=$ $n-\operatorname{rk} S$ (8). This last condition is the one expressed in (ii).

Then, from (9), $\mu^{-1}(0)$ has a single irreducible component of maximal dimension if and only if $\bmod \left(T, J_{I}\right)<\bmod \left(T, J_{\llbracket 1, n \rrbracket}\right)$ for any $I$ of the form $\llbracket 1, n \rrbracket \backslash\{i\}$. If $I=\llbracket 1, n \rrbracket \backslash\{i\}$, it follows from (8), that

$$
\bmod \left(T, J_{I}\right)=\left\{\begin{array}{ll}
\bmod \left(T, J_{\llbracket 1, n \rrbracket}\right)-1 & \text { if } \operatorname{rk} S_{\hat{\imath}}=\operatorname{rk} S \\
\bmod \left(T, J_{\llbracket 1, n \rrbracket}\right) & \text { if } \operatorname{rk} S_{\hat{\imath}}=\operatorname{rk} S-1
\end{array} .\right.
$$


In the next paragraph, we show that $\mu^{-1}(0)$ is a complete intersection, hence equidimensional. Together with the above arguments, this implies that (ii) and (iii) holds.

Consider the kernel of the representation $(T, V), K:=\left\{t \in T \mid \forall i, s_{i}(t)=1\right\}$. Its Lie algebra is $\mathfrak{k}:=\left\{t \in \mathfrak{t} \cong \mathbb{k}^{r} \mid S t=0\right\}=\operatorname{Ker}(S)$. Applying (7) to a general element $v \in V$, we get $\operatorname{dim}(T / K) \cdot v=\operatorname{dim} T \cdot v=\operatorname{rk} S=\operatorname{dim} \mathfrak{t}-\operatorname{dim} \mathfrak{k}$. So the representation $(T / K, V)$ is not only faithul but locally free. Note that $T / K$ is also a torus and that $\mu^{-1}(0)$ is the same for $(T, V)$ and $(T / K, V)$. Therefore, there is no loss of generality in assuming that $(T, V)$ is locally free in order to prove (i) and (iv). Under this assumption, important features are $\operatorname{rk} S=\operatorname{dim} T$ (7) and $\bmod (T, V)=\operatorname{dim} V-\operatorname{dim} T$ (10). In particular, we can apply Corollary 1.7 and get that $\mu^{-1}(0)$ is a complete intersection.

Assuming that $(T, V)$ is locally free, we claim that

$$
\forall I \subset \llbracket 1, n \rrbracket, \exists(x, \varphi) \in\left(\mu^{-1}(0)\right)_{J_{I}}, \mathfrak{t}^{(x, \varphi)}=\{0\} .
$$

Under this claim, (ii) and Corollary 1.7 (iii) imply that each irreducible component of $\mu^{-1}(0)$ has smooth points. Hence $\mu^{-1}(0)$ is reduced and this ends the proof of (i). The claim also implies that condition (5) is automatically satisfied so (iv) follows from Corollary $1.7(\mathrm{v})$.

Proof of claim (11) Let $I \subset \llbracket 1, n \rrbracket$. The decomposition $V=\bigoplus_{i} V_{i}$ induces to a decomposition $V^{*}=\bigoplus V_{i}^{*} \cong \mathbb{k}^{n}$. We set $x=v_{I}=\left(\delta_{i \in I}\right)_{i} \in J_{i} \subset V$ and $\varphi:=\left(\delta_{i \notin I}\right)_{i} \in V^{*}$ with respect to these decompositions. Since $\mathfrak{t} \cdot x \subset V_{I}$, we have $\varphi \in(\mathfrak{t} \cdot x)^{\perp}$ so $(x, \varphi) \in\left(\mu^{-1}(0)\right)_{J_{I}}$. Also, let $t \in T$ stabilizing $(x, \varphi)$. Then $s_{i}(t)=1$ for any $i \in I$ and $1 /\left(s_{i}(t)\right)=1$ for any $i \notin I$. Hence $t$ is in the kernel of the representation. By local freeness, we get $\mathfrak{t}^{x, \varphi}=\{0\}$.

\section{SympleCtiC REDUCTION FOR TORI REPRESENTATIONS}

We keep the notation and setting of the previous section. The aim of this section is to study the symplectic reduction $\left(V \oplus V^{*}\right) / / T:=\mu^{-1}(0) / / T$. We prove Conjecture 1.3 in the context of representations of tori (Corollary 3.10). Moreover, we show that that the conjecture is essentially false for non-visible action (Proposition 3.9), but that the symplectic reduction remains normal in this case (Proposition 3.4).

In this section, many properties depend on considerations on the convex hull of some of the weights $s_{i} \in X^{*}(T)$. If $\emptyset \neq A \subset X^{*}(T) \subset W_{\mathbb{Q}}=X^{*}(T) \otimes_{\mathbb{Z}} \mathbb{Q}$, we denote by $C H(A)$, the convex hull of $A$ in $W_{\mathbb{Q}}$. We also denote by ${ }^{\circ} H(A)$ the interior (for the classical topology) of $C H(A)$ in $\langle A\rangle$. Alternatively, $C^{\circ} H(A)$ is the set of convex combination $\sum_{a \in A} \alpha_{a} a$ with each $\alpha_{a}$ positive. It is an elementary result on convex hull that $0 \notin C H(A)$ (resp. $0 \notin C H(A)$ ) if and only if $\exists \phi \in W_{\mathbb{Q}}^{*}$ s. t. $\forall a \in A, \phi(a)>0$ (resp. $\phi(a) \geqslant 0$ and $\phi(A) \neq\{0\}$ ). Note that such $\phi$ can be multiplied by a sufficiently large integer so that we can always assume $\varphi \in X^{*}(T)$. In our context of weights on a group, this translates as follows. 
Lemma 3.1. Under above notation, $0 \notin C H(A)$ (resp. $0 \notin \stackrel{\circ}{C} H(A)$ ) if and only if there exists a one-parameter subgroup $\mathbb{C}^{\times} \stackrel{\rho}{\hookrightarrow} T$ such that $\forall a \in A, \exists b_{a} \in \mathbb{N}^{*}$ (resp. $b_{a} \in \mathbb{N}$ with at least one $a \in A$ s.t. $\left.b_{a} \neq 0\right), \forall t \in \mathbb{C}^{\times}, a(\rho(t))=t^{b_{a}}$.

Proposition 3.2. Let $\emptyset \neq I \subseteq \llbracket 1, n \rrbracket$ and $v \in J_{I}$.

(1) $v \in V$ is nilpotent if and only if $0 \notin C H\left(\left\{s_{i} \mid i \in I\right\}\right)$.

(2) $v \in V$ is semisimple if and only if $0 \in \stackrel{\circ}{C H}\left(\left\{s_{i} \mid i \in I\right\}\right)$.

Proof. First, recall that the elements of $J_{I}$ all share the same orbit dimension. In particular, an element $y \in \overline{T . v} \backslash T . v$ lies in $J_{I^{\prime}}$ for some $I^{\prime} \subsetneq I$. Then, from HilbertMumford criterion, $v=\left(v_{i}\right)_{i} \in V \backslash\{0\}$ is nilpotent (resp. is not semi-simple) if and only if there exists a one-parameter subgroup $\mathbb{C}^{\times} \stackrel{\rho}{\hookrightarrow} T$ such that $\rho(t) \cdot v_{i}=t^{b_{i}} v_{i}$ with $b_{i}$ positive for each $i \in I$ (resp. non-negative for each $i \in I$ and at least one $b_{i}$ non-zero) . Since $t^{b_{i}}=s_{i}(\rho(t))$, the result then follows from Lemma 3.1.

Given $(T, V)$ and associated weights $\left(s_{i}\right)_{i \in \llbracket 1, n \rrbracket}$, we split $\llbracket 1, n \rrbracket$ into

(12) $I_{d}:=\left\{i \in \llbracket 1, n \rrbracket \mid \operatorname{rk} S_{\hat{\imath}}=\operatorname{rk} S\right\}, \quad I_{f}:=I \backslash I_{d}=\left\{i \in \llbracket 1, n \rrbracket \mid \operatorname{rk} S_{\hat{\imath}}=\operatorname{rk} S-1\right\}$.

Corollary 3.3. The representation $(T, V)$ is stable if and only if $0 \in \stackrel{\circ}{\mathrm{C} H}\left(\left\{s_{i} \mid i \in\right.\right.$ $\llbracket 1, n \rrbracket\})$.

In this case $I_{f}=\emptyset$ and $\mu^{-1}(0)$ is irreducible and normal

Proof. A generic element of $V$ lies in $J_{\llbracket 1, n \rrbracket}$ so the first assertion follows from Proposition 3.2. If we can write 0 as a convex combination of the $\left(s_{i}\right)_{i \in \llbracket 1, n \rrbracket}$ with non-zero coefficients, $I_{f}$ is empty so the second statement follows from Theorem 2.2.

Proposition 3.4. There is an isomophism $\left(V \oplus V^{*}\right) / / T \cong\left(V_{I_{d}} \oplus V_{I_{d}}^{*}\right) / / T$. In particular, $\left(V \oplus V^{*}\right) / / T$ is normal.

Proof. We denote by $\mu_{I_{d}}$ the moment map $V_{I_{d}} \oplus V_{I_{d}}^{*} \rightarrow \mathfrak{t}^{*}$. Recall that $\mu^{-1}(0)$ and $\mu_{I_{d}}^{-1}(0)$ are reduced by Theorem 2.2. Hence so are $\left(V \oplus V^{*}\right) / / T=\mu^{-1}(0) / / T$ and $\left(V_{I_{d}} \oplus V_{I_{d}}^{*}\right) / / T=\mu_{I_{d}}^{-1}(0) / / T$. Note that $V_{I_{d}}^{*}$ can be identified with the subrepresentation of $V^{*}$ generated by the isotypical components associated to the weights $\left\{-s_{i} \mid i \in I_{d}\right\}$. This allows us to consider $\mu_{I_{d}}^{-1}(0)$ as a $T$-stable closed subscheme of $\mu^{-1}(0)$. Therefore, we have a closed immersion $\mu_{I_{d}}^{-1}(0) / / T \rightarrow \mu^{-1}(0) / / T$. To show that this is an isomorphism, there remains to prove that this map is a surjective. For this, we are going to show that the only closed orbits in $\mu^{-1}(0)$ lie in $\mu_{I_{d}}^{-1}(0)$.

Let $(x, y) \in \mu^{-1}(0)$ and assume that $x \notin V_{I_{d}}$. From the assumption on $x$, we know that there exists $i$ such that $x_{i} \neq 0$ and $\operatorname{rk} S_{\hat{\imath}}<\operatorname{rk} S$. Then there exists an element $t \in\left\langle s_{j} \mid j \neq i\right\rangle^{\perp} \subset \mathfrak{t}$ such that $s_{i}(t)=1$. So $t \cdot x=x_{i}$ and $V_{\{i\}} \subset \mathfrak{t} \cdot x$. Since $y \in(\mathfrak{t} \cdot x)^{\perp}$, we get $y_{i}=0$. Then a one-parameter subgroup $\mathbb{C}^{\times} \stackrel{\rho}{\rightarrow} T$ given by $s_{j}(\rho(t))=t^{\delta_{i, j}}$ acts on $(x, y)$ via $t \cdot y=y$ and $\lim _{t \rightarrow 0} t \cdot x=x^{\prime}$ with $x_{j}^{\prime}=x_{j}$ if $j \neq i$ and $x_{i}^{\prime}=0$. Hence $\left(x^{\prime}, y\right) \in \overline{T .(x, y)} \backslash T$. $(x, y)$. A similar argument shows that $T$. $(x, y)$ is also non-closed as soon as $y \notin V_{I_{d}}^{*}$.

Let us now show that $\left(V_{I_{d}} \oplus V_{I_{d}}^{*}\right) / / T$ is normal. For each $i \in I_{f}, s_{i}$ is not a linear combination of the other $s_{j}(j \neq i)$. Hence $\left\{s_{i} \mid i \in I_{f}\right\}$ induces a basis of $\left\langle s_{i}\right| i \in$ 
$\llbracket 1, n \rrbracket\rangle /\left\langle s_{i} \mid i \in I_{d}\right\rangle$ and $\operatorname{rk} S_{I_{d}}=\operatorname{rk} S-\# I_{f}$. Assuming that $\operatorname{rk} S_{I_{d} \backslash\{i\}}=\operatorname{rk} S_{I_{d}}-1$ for some $i \in I_{d}$, we would then have $\operatorname{rk} S_{\hat{\imath}} \leqslant \operatorname{rk} S_{I_{d}}-1+\#\left(I_{f}\right)=\operatorname{rk} S-1$, which contradicts the assumption $i \in I_{d}$. So rk $S_{I_{d} \backslash\{i\}}=\operatorname{rk} S_{I_{d}}$ for any $i \in I_{d}$ and $\left(T, V_{I_{d}}\right)$ satisfies hypothesis of Theorem 2.2 (iii-iv). Hence $\mu_{I_{d}}^{-1}(0)$ is irreducible and normal, so the same holds for its quotient by $T$.

We have the following useful characterization of a visible action:

Proposition 3.5. The representation $(T, V)$ with weight $\left(s_{i}\right)_{i \in \llbracket 1, n \rrbracket}$ is visible if and only if there is a partition $\llbracket 1, n \rrbracket=\bigsqcup_{j=0}^{l} I_{j}$ satisfying

(i) $\left\langle s_{i} \mid i \in \llbracket 1, n \rrbracket\right\rangle=\bigoplus_{j=0}^{l}\left\langle s_{i} \mid i \in I_{j}\right\rangle$

(ii) $\operatorname{dim}\left\langle s_{i} \mid i \in I_{j}\right\rangle=\left\{\begin{array}{l}\# I_{j} \text { if } j=0 \\ \# I_{j}-1 \text { if } j \geqslant 1\end{array}\right.$

(iii) $\forall j \geqslant 1,0 \in \stackrel{\circ}{C H}\left(\left\{s_{i} \mid i \in I_{j}\right\}\right)$.

Note that the subset $I_{0}$ in the proposition has to coincide with $I_{f}$ defined in (12) and that $I_{d}=\bigsqcup_{j \geqslant 1} I_{j}$.

Proof. "if" part: Assume that such a partition exists. Let $x \in V$ be nilpotent and let $\tilde{I}$ be such that $x \in J_{\tilde{I}}$. Define $\tilde{I}_{j}:=\tilde{I} \cap I_{j}$. Assume that $j$ is a positive index such that $\tilde{I}_{j} \neq \emptyset$. Since $x$ is nilpotent and $\tilde{I}_{j} \subset \tilde{I}$, it follows from Proposition 3.2 that $0 \notin C H\left(\left\{s_{i} \mid i \in \tilde{I}_{j}\right\}\right)$. By (iii), $\tilde{I}_{j}$ is a proper subset of $I_{j}$.

On the other hand, (iii) also implies that there is a convex combination $\sum_{i \in I_{j}} \alpha_{i} s_{i}=$ 0 with each $\alpha_{i}>0$. By (ii), this combination is the unique linear relation (up to scalar multiplication) between the $\left(s_{i}\right)_{i \in I_{j}}$. In particular, the family $\left(s_{i}\right)_{i \in \tilde{I}_{j}}$ is linearly independant. The same hold if $j=0$ by (ii). By (i), we get that $\left(s_{i}\right)_{i \in \tilde{I}}$ is linearly independent.

Then, by $(7), \operatorname{dim} T . x=\operatorname{rk} S_{\tilde{I}}=\# \tilde{I}=\operatorname{dim} J_{\tilde{I}}$. Hence T. $x$ is open in $J_{\tilde{I}}$. Since this holds for any $x \in J_{\tilde{I}}$, we get $T . x=J_{\tilde{I}}$. Since there are finitely many possible index set $\tilde{I}$, the representation $(T, V)$ is visible.

"only if" part: We will argue by induction on $\operatorname{dim} V$. Assume $\operatorname{dim} V=1$. If $s_{1} \neq 0$, we set $l=0$ and $I_{0}:=\left\{s_{1}\right\}$. If $s_{1}=0$, we set $l:=1, I_{0}:=\emptyset$ and $I_{1}:=\left\{s_{1}\right\}$.

Assume now that the result holds true for any visible representation of $T$ of dimension $n-1$ and consider a visible representation $(T, V)$ of dimension $n$. Since $V^{\prime}:=V_{\llbracket 1, n-1 \rrbracket}$ is a subrepresentation of $V$, it is also visible. By the inductive hypothesis, there exists a partition $\llbracket 1, n-1 \rrbracket=\bigsqcup_{j=0}^{l^{\prime}} I_{j}^{\prime}$ satisfying (i), (ii) and (iii). If $s_{n} \notin\left\langle s_{i} \mid i \in \llbracket 1, n-1 \rrbracket\right\rangle$, we can set $I_{0}:=I_{0}^{\prime} \cup\{n\}$ and $I_{j}:=I_{j}^{\prime}$ for any $j \geqslant 1$.

From now on, we assume that $s_{n} \in\left\langle s_{i} \mid i \in \llbracket 1, n-1 \rrbracket\right\rangle$. As a first step, we claim that there is a subset $\tilde{I} \subset \llbracket 1, n-1 \rrbracket$ and some non-zero rational coefficients $\left(\beta_{i}\right)_{i \in \tilde{I}}$ such that

- $s_{n}:=\sum_{i \in \tilde{I}} \beta_{i} s_{i}$

- $\tilde{I} \cap I_{j}^{\prime} \neq I_{j}^{\prime}$ for any $j \geqslant 1$

- $\beta_{i}>0$ for $i \in \bigsqcup_{j \geqslant 1} I_{j}^{\prime} \cap \tilde{I}$.

Indeed, start with a combination with rational coefficients $s_{n}:=\sum_{i \in \llbracket 1, n-1 \rrbracket} \beta_{i}^{\prime} s_{i}$. Fix $j \geqslant 1$. From (iii) and (ii), there is a combination with positive coefficients 
$0=\sum_{i \in I_{j}^{\prime}} \alpha_{i} s_{i}$. Let $\lambda_{j}:=\max _{i \in I_{j}^{\prime}}\left\{-\beta_{i}^{\prime} / \alpha_{i}\right\}$. Set $\beta_{i}:=\beta_{i}^{\prime}+\lambda_{j} \alpha_{i}$ for $i \in I_{j}^{\prime}$. So $\beta_{i} \geqslant 0$ for any $i \in I_{j}^{\prime}$ and $\beta_{i}=0$ for at least one $i \in I_{j}^{\prime}$. Then set $\tilde{I}:=\left\{i \mid \beta_{i} \neq 0\right\}$ and the claim is shown.

From the property $\tilde{I} \cap I_{j}^{\prime} \neq I_{j}^{\prime}$ and (i), (ii), (iii), we get that $\left(s_{i}\right)_{i \in \tilde{I}}$ is linearly independant as in the proof of the "if part". So, up to scalar multiplication, the only relation between $\left\{s_{i} \mid i \in \tilde{I} \cup\{n\}\right\}$ is given by the above coefficients $\beta_{i}(i \in \tilde{I})$. Assuming that $\beta_{i}>0$ for some $i \in \tilde{I}$ (e.g. if $\tilde{I} \cap \bigsqcup_{j \geqslant 1} I_{j}^{\prime} \neq \emptyset$ ), we get $0 \notin C H\left(\left\{s_{i} \mid i \in\right.\right.$ $\tilde{I} \cup\{n\}\})$ so orbits in $J_{\tilde{I} \cup\{n\}}$ are nilpotent by Proposition 3.2. From (7), these orbits are of dimension $\operatorname{rk} S_{\tilde{I} \cup\{n\}}=\# \tilde{I}=\operatorname{dim} J_{\tilde{I} \cup\{n\}}-1$. This contradicts the visibility of $(T, V)$. As a result, $\tilde{I} \subset I_{0}$ and $\beta_{i}<0$ for each $i \in I_{0}$. Setting $l:=l^{\prime}+1$, $I_{l}:=\tilde{I} \cup\{n\}, I_{0}:=I_{0}^{\prime} \backslash \tilde{I}$ and $I_{j}:=I_{j}^{\prime}$ for $j \in \llbracket 1, l^{\prime} \rrbracket$ then yield (i), (ii) and (iii) for the representation $(T, V)$.

Remark 3.6. We can re-interpret Proposition 3.5 as follows. The visible tori actions are essentially made of two part: a part of rank 0 provided by $I_{0}$ and several stable blocks of rank 1, each looking very much like [BLLT, Example 8.6]. There are other appearence of stable blocks of rank 1 in Lemma 4.9.

Corollary 3.7. Any visible representation of a torus is polar.

Proof. Let $(T, V)$ be a visible representation and adopt notation of Proposition 3.5. For $\underline{w}:=\left(w_{1}, \ldots, w_{l}\right) \in \mathbb{C}^{l}$, we consider $x(\underline{w})$ as the element $\left(x_{i}\right)_{i \in \llbracket 1, n \rrbracket}$ given by $x_{i}:=0$ if $i \in I_{0}$ and $x_{i}:=w_{j}$ if $i \in I_{j}(j \geqslant 1)$. From (6), we get $\mathfrak{t} \cdot x(\underline{w})=$ $X_{x(\underline{w})} \operatorname{Im}(S)$. From Proposition $3.5(\mathrm{i}), \operatorname{Im}(S)=\bigoplus_{j} \operatorname{Im}\left(S_{I_{j}}\right)$. Since $X_{x(\underline{w})}$ acts by $w_{j} \operatorname{Id}$ on $\operatorname{Im}\left(S_{I_{j}}\right)$, we get that $\mathfrak{t} \cdot x(\underline{w})$ is included in $\mathfrak{t} \cdot x(1, \ldots, 1)$. The subspace $\mathfrak{c}:=\left\{x(\underline{w}) \mid \underline{w} \in \mathbb{C}^{l}\right\}$ is our candidate for being a Cartan subspace of $(T, V)$ and there remains to show that $l=\operatorname{dim} V / / T$, [DK].

Since $(T, V)$ is visible, a general fiber of the quotient map $V \rightarrow V / / T$ is the closure of a general orbit in $V$. Hence, by $(7), \operatorname{dim} V / / T=\operatorname{dim} V-\operatorname{rk} S$. This is equal to $l$ by Proposition 3.5.

Let us now take a closer look at $\left(V \oplus V^{*}\right) / / / T$, that is at closed orbits of $\mu^{-1}(0)$. A first observation is the following.

Lemma 3.8. If $(x, y) \in \mu^{-1}(0)$ is such that $x \in V$ and $y \in V^{*}$ are semisimple, then $(x, y)$ is semisimple in $V \oplus V^{*}$.

Proof. Consider the set of weigths for the action of $T$ on $V \oplus V^{*}$. Denote by $A_{x}$ (resp. $A_{y}, A_{x, y}$ ) the set of weights corresponding to the support of $x$ (resp. $\left.y,(x, y)\right)$. By Proposition 3.2, $0 \in \stackrel{\circ}{C} H\left(A_{x}\right)$ and $0 \in \stackrel{\circ}{C} H\left(A_{y}\right)$. So $0 \in \stackrel{\circ}{C} H\left(A_{x} \cup A_{y}\right)=\stackrel{\circ}{C} H\left(A_{x, y}\right)$. The result follows.

Proposition 3.9. The representation $(T, V)$ is visible if and only if closed orbits of $\mu^{-1}(0)$ are those of the form T.(x,y) with T.x and T.y closed.

Proof. - Assume that $(T, V)$ is visible. With similar arguments to those of Proposition 3.4, we are going to show that, whenever $x \in V$ is not semisimple, $(x, y) \in$ 
$\mu^{-1}(0)$ cannot be semisimple. A symmetric argument on $y \in V^{*}$ and Lemma 3.8 then allow to conclude for the "only if" part.

Let $x \in J_{\tilde{I}}$ for some $\tilde{I} \subset \llbracket 1, n \rrbracket$. The representation $\left(T, V_{\tilde{I}}\right)$ is also visible, so we can apply Proposition 3.5 (with $\tilde{I}$ instead of $\llbracket 1, n \rrbracket)$ and get a partition $\tilde{I}=\bigsqcup_{j=0}^{l} \tilde{I}_{j}$. Apply also Proposition 3.5 to $V$, so that we get a partition $\llbracket 1, n \rrbracket=\bigsqcup_{j} I_{j}$.

Assuming that $x$ is not semisimple amounts to assume that $\tilde{I}_{0} \neq \emptyset$ (Proposition 3.2 ). If $\tilde{I}_{0} \cap I_{0} \neq \emptyset$, Proposition 3.4 states that any element of $\mu^{-1}(0)$ of the form $(x, y)$ is not semisimple. From now on, we assume that there exists $j \geqslant 1$ such that $\tilde{I}_{0} \cap I_{j} \neq \emptyset$. From property (ii) of Proposition 3.5 on $\tilde{I}_{0}$ and $I_{j}$, we also know that $I_{j} \backslash \tilde{I}_{0} \neq \emptyset$. Fix $i_{0} \in \tilde{I}_{0} \cap I_{j}$ and $i_{1} \in I_{j} \backslash \tilde{I}_{0}$. The relation between the $\left(s_{i}\right)_{i \in I_{j}}$ can be written as $\sum_{i \in I_{j}} \alpha_{i} s_{i}=0$ with the $\alpha_{i}$ positive integers. Then we can define a one parameter subgroup $\rho$ : $\mathbb{C}^{\times} \hookrightarrow T$, via

$$
s_{i}(\rho(t))= \begin{cases}t^{\alpha_{i_{1}}} & \text { if } i=i_{0} \\ t^{-\alpha_{i_{0}}} & \text { if } i^{\prime}=i_{1} \\ 1 & \text { else }\end{cases}
$$

Then $V_{\left\{i_{0}\right\}}=\operatorname{Lie}\left(\rho\left(\mathbb{C}^{\times}\right)\right) \cdot x \subset \mathfrak{t} \cdot x$ and, whenever $(x, y) \in \mu^{-1}(0)$, we have $y_{i_{0}}=0$. Since $\rho(t)$ acts on $y_{i_{1}}$ by multiplication by $t^{\alpha_{i}}$, we get that $\lim _{t \rightarrow 0}(\rho(t) \cdot(x, y))$ exists and that its first component lies in $J_{\tilde{I} \backslash\left\{i_{0}\right\}}$. In particular, T. $(x, y)$ is not closed.

- Assume now that $(T, V)$ is not visible. We want to find $(x, y)$ semisimple such that $x$ is not semisimple. Let $I \subset \llbracket 1, n \rrbracket$ be such that $J_{I}$ contains infinitely many nilpotent orbits. Then $0 \notin C H\left(\left\{s_{i} \mid i \in I\right)\right.$ (Proposition 3.2) and $\operatorname{rk} S_{I}<$ $\operatorname{dim} J_{I}=\# I(7)$. Thus there exists non-trivial linear relations $\sum_{i \in I} \alpha_{i} s_{i}=0$ with integer coefficients and for any such relation the $\alpha_{i}$ are not all of the same sign. We can therefore write a relation of the form $\sum_{i \in I^{\prime}} \beta_{i} s_{i}=\sum_{i \in I^{\prime \prime}} \beta_{i} s_{i}$ with each $\beta_{i}$ positive, $I^{\prime}, I^{\prime \prime} \neq \emptyset$ and $I^{\prime} \sqcup I^{\prime \prime} \subset I$. Let $x=\left(x_{i}\right)_{i} \in J_{I^{\prime}}$ and, analogously in $V^{*}$, let $y=\left(y_{i}\right)_{i} \in J_{I^{\prime \prime}} \subset V_{I^{\prime \prime}}^{*} \subset V^{*}$. From this, we get that $x$ is non-zero nilpotent, that $y \in V_{I^{\prime \prime}}^{*} \subset\left(V_{I^{\prime}}\right)^{\perp} \subset(\mathfrak{t} \cdot x)^{\perp}$ and that

$$
T .(x, y) \subset\left\{\left(x^{\prime}, y^{\prime}\right) \in V_{I^{\prime}} \times V_{I^{\prime \prime}}^{*} \mid \prod_{i \in I^{\prime}}\left(x_{i}^{\prime}\right)^{\beta_{i}} \prod_{i \in I^{\prime \prime}}\left(y_{i}^{\prime}\right)^{\beta_{i}}=\prod_{i \in I^{\prime}}\left(x_{i}\right)^{\beta_{i}} \prod_{i \in I^{\prime \prime}}\left(y_{i}\right)^{\beta_{i}}\right\} .
$$

This last subset is a $T$-invariant closed subset of $V$ which does not intersect $\{0\} \times V^{*}$. Let $\left(x^{\prime}, y^{\prime}\right)$ be in the closed orbit of $\overline{T .(x, y)}$. Since $x^{\prime} \subset J_{\tilde{I}}$ with $\tilde{I} \subset I^{\prime}, x^{\prime}$ is non-zero nilpotent hence not semisimple.

Proposition 3.9 shows that the visibility assumption in Conjecture 1.3 is necessary, since the statement fails set-theoretically in any non-visible case.

When $\mathbb{k}=\mathbb{C}$, we can state the following:

Corollary 3.10. Conjecture 1.3 holds true for representations of tori

Proof. Note that the polar assumption is redundant by Corollary 3.7. According to Proposition 3.9, the generic elements of the irreducible (Proposition 3.4) variety $V \oplus V^{*} / / T$ are closed orbits of the form $T .(x, y)$ with $x$ regular (i.e. with T.x closed of maximal dimension among closed orbits). We may assume that $x \in \mathfrak{c}$. 
Then $y \in(\mathfrak{t} \cdot x)^{\perp}=(\mathfrak{t} \cdot \mathfrak{c})^{\perp}=\mathfrak{c}^{\vee} \oplus U^{\vee}$ and, since T.y is closed, we get $y \in \mathfrak{c}^{\vee}$ [DK, Corollary 2.5]. Hence (1) of Remark 1.4 holds Statement (2) follows from Proposition 3.4 .

\section{Null-Fiber of the MOMENT MAP - $\theta$ CASE}

A standard reference for the theory of $\theta$-representations is [Vin]. These representations are visible and polar but they are not always stable. A classification of the irreducible $\theta$-representations with their main features can be found in [Kac, §3].

Let us first introduce some notation. Let $\mathfrak{h}=\bigoplus_{i \in \mathbb{Z}_{m}} \mathfrak{h}_{i}$ be a reductive Lie algebra equipped with a $\mathbb{Z}_{m}$-grading, that is $\left[\mathfrak{h}_{i}, \mathfrak{h}_{j}\right] \subset \mathfrak{h}_{i+j}$. Let $H$ be any connected algebraic group having $\mathfrak{h}$ as its Lie algebra. We consider the adjoint representation of $G:=H_{0}$ on $V:=\mathfrak{h}_{1}$ where $H_{0}$ is the connected subgroup of $H$ with Lie algebra $\mathfrak{h}_{0}$. Fix $\omega$ an $m$-th root of 1 . We denote by $\theta$ the automorphism of $\mathfrak{h}$ given by $\theta(x)=\omega^{i} x$ for $x \in \mathfrak{h}_{i}$. If $\mathfrak{a}$ is a $\theta$-stable subspace of $\mathfrak{h}$, we can decompose $\mathfrak{a}=\bigoplus \mathfrak{a}_{i}$ with $\mathfrak{a}_{i}:=\mathfrak{a} \cap \mathfrak{h}_{i}$. We then denote the dual direct sum by $\mathfrak{a}^{*}=\bigoplus \mathfrak{a}_{i}^{\vee}$. We will identify $\mathfrak{a}_{i}^{\vee}$ and $\left(\mathfrak{a}_{i}\right)^{*}$.

We need to understand $\mu^{-1}(0)$. From [Vin, $\S 1.2$ and $\S 2.2$ ], the $\theta$-representation $\left(H_{0}, \mathfrak{h}_{1}\right)$ is isomorphic to $\left(\prod_{k}\left(H^{(k)}\right)_{0}, \prod\left(\mathfrak{h}^{(k)}\right)_{1}\right)$ for some graded Lie algebras $\mathfrak{h}^{(k)}=$ $\bigoplus\left(\mathfrak{h}^{(k)}\right)_{i}$ which are either simple or abelian. The $\left(\left(H^{(k)}\right)_{0},\left(\mathfrak{h}^{(k)}\right)_{1}\right)$ with $\mathfrak{h}$ simple are called the simple components of $\left(H_{0}, \mathfrak{h}_{1}\right)$. Then $\mu^{-1}(0)$ is the direct product of the $\mu_{k}^{-1}(0)$ where $\mu_{k}$ is the moment map associated to the representation $\left(\left(H^{(k)}\right)_{0},\left(\mathfrak{h}^{(k)}\right)_{1}\right)$. In the abelian case, $\mu^{-1}(0)=\mathfrak{h}_{1} \oplus \mathfrak{h}_{1}^{*}$. From now on, we will assume that $\mathfrak{h}$ is simple.

The Killing form $L(\cdot, \cdot)$ is a non-degenerate symmetric bilinear form on $\mathfrak{h} \times \mathfrak{h}$ which is invariant under $H$ and $\theta$. Via $L, V^{*} \cong \mathfrak{h}_{1}^{\vee}$ identifies to $\mathfrak{h}_{-1}$. If $x \in \mathfrak{h}$ and $\mathfrak{a} \subset \mathfrak{h}$, we denote the centraliser of $x$ in $\mathfrak{a}$ by $\mathfrak{a}^{x}=\{y \in \mathfrak{a} \mid[x, y]=0\}$. Assume that $\varphi \in V^{*}$ corresponds to $y \in \mathfrak{h}_{-1}$ via $L$. Then the condition $\varphi \in(\mathfrak{g} \cdot x)^{\perp}$ is read $0=L([\mathfrak{g}, x], y)=L\left(\mathfrak{h}_{0},[x, y]\right)$, that is $[x, y]=0$ since $[x, y] \in \mathfrak{h}_{0}$ and $L$ is non-degenerate on $\mathfrak{h}_{0} \oplus \mathfrak{h}_{0}$. Thus $\mu^{-1}(0)$ is isomorphic to the commuting scheme:

$$
\mu^{-1}(0) \cong\left\{(x, y) \in \mathfrak{h}_{1} \times \mathfrak{h}_{-1} \mid[x, y]=0\right\} .
$$

We now want to define a suitable locally closed cover of $\mathfrak{h}_{1}$ satisfying the conditions stated in Section 1

Definition 4.1. We say that a Levi subalgebra $\mathfrak{l}$ of $\mathfrak{h}$ arises from $\mathfrak{h}_{1}$ if there exists a semisimple element $s \in \mathfrak{h}_{1}$ such that $\mathfrak{l}=\mathfrak{h}^{s}$.

In other words, $(\mathfrak{z} \mathfrak{l})_{\text {reg }} \cap \mathfrak{h}_{1} \neq \emptyset$ where $\mathfrak{z} \mathfrak{r}$ is the center of $\mathfrak{l}$ and $(\mathfrak{z} \mathfrak{l})_{\text {reg }}$ denotes the set of elements of $\mathfrak{z} \mathfrak{r}$ whose $H$-orbit is of maximal dimension. Such a Levi $\mathfrak{l}$ is clearly $\theta$-stable. Hence $\mathfrak{l}=\bigoplus \mathfrak{l}_{i}$ gives rise to a $\mathbb{Z}_{m}$-graded reductive Lie algebra. We denote by $L_{0}$ the connected subgroup of $H$ with Lie algebra $\mathfrak{l}_{0}$.

Recall from [Vin] that any $x \in \mathfrak{h}_{1}$ has a Jordan decomposition $x_{s}+x_{n}$ with $x_{s}, x_{n} \in \mathfrak{h}_{1}$, the semisimple and nilpotent part of $x$, respectively. 
Definition 4.2. Given $\mathfrak{l}$ a Levi subalgebra of $\mathfrak{h}$ arising from $\mathfrak{h}_{1}$, and $\mathcal{O}$ a nilpotent $L_{0}$-orbit in $\mathfrak{l}_{1}$, we define the decomposition class associated to $(\mathfrak{l}, \mathcal{O})$ as

$$
J(\mathfrak{l}, \mathcal{O}):=H_{0} \cdot\left\{(\mathfrak{z} \mathfrak{l})_{\text {reg }} \cap \mathfrak{h}_{1}+\mathcal{O}\right\}
$$

Alternatively, $x \in J(\mathfrak{l}, \mathcal{O})$ if and only if $x$ is $H_{0}$-conjugate to an element $x^{\prime} \in \mathfrak{l}_{1}$ such that $\mathfrak{l}=\mathfrak{h}^{x_{s}^{\prime}}$ and $\mathcal{O}=L_{0} \cdot x_{n}^{\prime}$. Hence decomposition classes are equivalence classes, so they form a partition of $\mathfrak{h}_{1}$. Next, we adapt some results of [Br] (see also [TY, §39]).

Proposition 4.3. Let $J(\mathfrak{l}, \mathcal{O})$ be a decomposition class.

(i) If $x \in J(\mathfrak{l}, \mathcal{O})$, then $J(\mathfrak{l}, \mathcal{O})=H_{0} \cdot\left\{y \in \mathfrak{h}_{1} \mid \mathfrak{h}^{y}=\mathfrak{h}^{x}\right\}$

(ii) $J(\mathfrak{l}, \mathcal{O})$ is an irreducible locally closed subset of $\mathfrak{h}_{1}$.

(iii) If $x \in J(\mathfrak{l}, \mathcal{O})$ then $\operatorname{dim} H_{0} . x=\operatorname{dim} \mathfrak{h}_{1}-\operatorname{dim} \mathfrak{l}_{1}+\operatorname{dim} \mathcal{O}$.

(iv) $\bmod \left(H_{0}, J(\mathfrak{l}, \mathcal{O})\right)=\operatorname{dim}(\mathfrak{z} \mathfrak{l})_{1}$.

Proof. There is no loss of generality in assuming that $x$ satisfies $\mathfrak{h}^{x_{s}}=\mathfrak{l}$ and $\mathcal{O}=$ $L_{0} \cdot x_{n}$.

(i) From [TY, 35.3.3, 39.1.2, 39.1.1], we have $\left\{y \in \mathfrak{h}_{1} \mid \mathfrak{h}^{y}=\mathfrak{h}^{x}\right\}=\left\{y \in \mathfrak{l}_{1} \mid \mathfrak{h}^{y_{s}}=\right.$ $\left.\mathfrak{l},\left(\mathfrak{l}^{\prime}\right)^{y_{n}}=\left(\mathfrak{l}^{\prime}\right)^{x_{n}}\right\}$ where $\mathfrak{l}^{\prime}:=[\mathfrak{l}, \mathfrak{l}]$. Since $L_{0}$ acts trivially on $(\mathfrak{z} \mathfrak{l})_{\text {reg }}$, this yields $J(\mathfrak{l}, \mathcal{O}) \subset H_{0} \cdot\left\{y \in \mathfrak{h}_{1} \mid \mathfrak{h}^{y}=\mathfrak{h}^{x}\right\}$. Note that the condition $\mathfrak{h}^{y_{s}}=\mathfrak{l}$ implies that $y, y_{s}, y_{n} \in \mathfrak{l}_{1}$. In order to prove the converse inclusion, it is therefore enough to show that $\mathcal{U}:=\left\{n \in \mathfrak{l}_{1}^{\prime} \mid\left(\mathfrak{l}^{\prime}\right)^{n}=\left(\mathfrak{l}^{\prime}\right)^{x_{n}}\right\} \subset \mathcal{O}$. Let $n \in \mathcal{U}$. Then $\mathfrak{l}_{0}^{n}=\mathfrak{l}_{0}^{x_{n}}$ so $\operatorname{dim} L_{0} \cdot n=\operatorname{dim} L_{0} \cdot x_{n}=\operatorname{dim} \mathcal{O}$. The subset $\mathcal{U}$ is a dense open subset of $\left(\mathfrak{l}_{1}^{\prime}\right)^{x_{n}}$ by [TY, 35.3.3], hence $\mathcal{U}$ is irreducible. From [TY, 35.3.4], $\mathcal{U}$ is included in the nilpotent cone of $\mathfrak{l}_{1}^{\prime}$ which consists of fintely many $L_{0^{-}}$orbits. Let $\mathcal{O}^{\prime}$ be the $L_{0^{-}}$ nilpotent orbit whose intersection with $\mathcal{U}$ is dense in $\mathcal{U}$. Then $\mathcal{U} \subset \overline{\mathcal{O}^{\prime}}$. Since all the elements of $\mathcal{U}$ share the same $L_{0}$-orbit dimension and $x_{n} \in \mathcal{O} \cap \mathcal{U}$, we can eventually conclude that $\mathcal{O}=\mathcal{O}^{\prime}$ and $\mathcal{U} \subset \mathcal{O}$.

(ii) It is clear from the definition that $J(\mathfrak{l}, \mathcal{O})$ is irreducible. Set $m=\operatorname{dim} \mathfrak{h}^{x}$ for some $x \in J(\mathfrak{l}, \mathcal{O})$. By (i), it does not depend on the choice of $x$. The map $\varphi: y \mapsto \mathfrak{h}^{y}$, from $\left\{y \in \mathfrak{h} \mid \operatorname{dim} \mathfrak{h}^{y}=m\right\}$ to the Grassmannian of $m$-dimensional subspaces in $\mathfrak{h}$, is a morphism of varieties [TY, 19.7.6, 29.3.1]. By $(\mathrm{i}), J(\mathfrak{l}, \mathcal{O})=\mathfrak{h}_{1} \cap \varphi^{-1}\left(H_{0} \cdot\left(\mathfrak{h}^{x}\right)\right)$ with $\mathfrak{h}^{x}$ seen as an element of the Grassmannian. As orbits are locally closed sets, so are their inverse images by morphisms of varieties. Hence $J(\mathfrak{l}, \mathcal{O})$ is locally closed in $\left\{y \in \mathfrak{h} \mid \operatorname{dim} \mathfrak{h}^{y}=m\right\}$ and the result follows.

(iii) Consider ad $x$ as an endomorphism of $\mathfrak{h}$. Then $\mathfrak{l}$ is its generalized eigenspace associated to 0 . Let us denote by $\mathfrak{q}$ the sum of the other generalized eigenspaces. We have $\mathfrak{h}=\mathfrak{l} \oplus \mathfrak{q}$ and $\mathfrak{h} \cdot x=[\mathfrak{l} \oplus \mathfrak{q}, x]=(\operatorname{ad} x)(\mathfrak{l}) \oplus(\operatorname{ad} x)(\mathfrak{q})=\left(\operatorname{ad} x_{n}\right)(\mathfrak{l}) \oplus \mathfrak{q}$. Intersect with $\mathfrak{h}_{1}$ yields $\mathfrak{h}_{0} \cdot x=\mathfrak{l}_{0} \cdot x_{n} \oplus \mathfrak{q}_{1}$. In particular, $\operatorname{codim}_{\mathfrak{h}_{1}} H_{0} \cdot x=\operatorname{codim}_{\mathfrak{l}_{1}} L_{0} \cdot x_{n}$.

(iv) From the computations in (iii), we also get that $\mathfrak{h} \cdot x$ has trivial intersection with $\mathfrak{z}$. Since $\mathfrak{h}_{0} \cdot x+(\mathfrak{z} \mathfrak{l})_{1}$ is the tangeant space of $J(\mathfrak{l}, \mathcal{O})$ at $x$, we get $\operatorname{dim} J(\mathfrak{l}, \mathcal{O})=\operatorname{dim} H_{0} \cdot x+\operatorname{dim}(\mathfrak{z} \mathfrak{l})_{1}$. Since the elements of $J(\mathfrak{l}, \mathcal{O})$ all share the same orbit dimension, we have $\bmod \left(H_{0}, J(\mathfrak{l}, \mathcal{O})\right)=\operatorname{dim} J(\mathfrak{l}, \mathcal{O})-\operatorname{dim} H_{0} \cdot x$. The result follows. 
From Proposition 4.3, the decomposition classes satisfy the requirements of the covers used in Section 1. However, note that two different data $\left(\mathfrak{l}_{i}, \mathcal{O}_{i}\right), i=1,2$ can give rise to the same decomposition class. In the sequel, we fix a Cartan subspace $\mathfrak{c} \subset \mathfrak{h}_{1}$, that is a maximal subspace of commuting semisimple elements. The number $\operatorname{dim} \mathfrak{c}$ is called the rank of $\left(H_{0}, \mathfrak{h}_{1}\right)$.

Definition 4.4. We say that a property $\mathcal{P}$ is hereditary if, whenever $\mathcal{P}$ holds on a $\theta$-representation $\left(H_{0}, \mathfrak{h}_{1}\right)$, it holds on the restricted $\theta$-representation $\left(\left(H_{0}^{s}\right)^{\circ}, \mathfrak{h}_{1}^{t}\right)$ for any semisimple element $t \in \mathfrak{h}_{1}$. Here, $\left(H_{0}^{s}\right)^{\circ}$ is the connected subgroup of $H_{0}$ whose Lie algebra is $\mathfrak{g}_{0}^{s}$.

Next, we recall a few classical facts.

Lemma 4.5. Let $\left(H_{0}, \mathfrak{h}_{1}\right)$ be a $\theta$-representation with Cartan subspace $\mathfrak{c} \subset \mathfrak{h}_{1}$.

(i) For an element in general position $s \in \mathfrak{c}$, we have $\mathfrak{h}^{s}=\mathfrak{h}^{\mathfrak{c}}$ and $\left(\mathfrak{z}^{(}\left(\mathfrak{h}^{s}\right)\right)_{1}=\mathfrak{c}$.

(ii) $\left(H_{0}, \mathfrak{h}_{1}\right)$ is locally free if and only if $\operatorname{dim} \mathfrak{h}_{1}=\operatorname{dim} \mathfrak{h}_{0}+\operatorname{dim} \mathfrak{c}$.

(iii) $\left(H_{0}, \mathfrak{h}_{1}\right)$ is stable if and only if there exists $s \in \mathfrak{c}$ such that $\mathfrak{h}_{1}^{s}=\mathfrak{z}\left(\mathfrak{h}^{s}\right)_{1}=\mathfrak{c}$.

(iv) Stability and local freeness are both hereditary properties.

Proof. (i) follows from [Vin, §3.2].

Since $\theta$-representations are visible, it follows from Remark 1.8 that $\bmod \left(H_{0}, \mathfrak{h}_{1}\right)=$ $\operatorname{dim} \mathfrak{h}_{1}-m_{0}$ where $m_{0}$ is the maximal orbit dimension in $\mathfrak{h}_{1}$. By (i), Proposition 4.3 (iv) and (1), the maximal modality of a decomposition class is $\bmod \left(H_{0}, \mathfrak{h}_{1}\right)=\operatorname{dim} \mathfrak{c}$. Assertion (ii) follows.

From Proposition 4.3 (iii), an element $x=x_{s}+x_{n}$ can have maximal orbit dimension in $\mathfrak{h}_{1}$ only if $L_{0} \cdot x_{n}$ is of maximal dimension in $\mathfrak{l}_{1}$ with $\mathfrak{l}=\mathfrak{h}^{x_{s}}$. This is possible with $x_{n}=0$ only if $\mathfrak{l}_{1}^{\prime}=\{0\}$ where $\mathfrak{l}^{\prime}=[\mathfrak{l}, \mathfrak{l}]$. Since $\mathfrak{l}_{1}$ contains a Cartan subspace, this implies that $\mathfrak{l}_{1}$ is a Cartan subspace. On the other hand, assume that there exists $s \in \mathfrak{c}$ such that $\mathfrak{h}_{1}^{s}=\mathfrak{z}\left(\mathfrak{h}^{s}\right)_{1}=\mathfrak{c}$. Then $J\left(\mathfrak{h}^{s},\{0\}\right)$ is a decomposition class of $\operatorname{dimension} \operatorname{dim} H_{0} \cdot s+\bmod \left(H_{0}, J\left(\mathfrak{h}^{s},\{0\}\right)\right)=\operatorname{dim} \mathfrak{h}_{1}-\operatorname{dim} \mathfrak{h}_{1}^{s}+0+\operatorname{dim} \mathfrak{z}\left(\mathfrak{h}^{s}\right)_{1}=$ $\operatorname{dim} \mathfrak{h}_{1}$ by Proposition 4.3. Hence $J\left(\mathfrak{h}^{s},\{0\}\right)$ is the open class in $\mathfrak{h}_{1}$. Since it is made of semisimple elements, the representation is stable.

(iv) follows from [BLLT, Lemma 6.3].

Recall from [BLLT, Proposition 4.3] that $\mu^{-1}(0)$ can be irreducible only if $\left(H_{0}, \mathfrak{h}_{1}\right)$ is stable.

Theorem 4.6. Assume that $\left(H_{0}, \mathfrak{h}_{1}\right)$ is a stable and locally free $\theta$-representation with $\mathfrak{h}$ simple, then

i) $\mu^{-1}(0)$ is an irreducible and reduced complete intersection

ii) $\mu^{-1}(0)$ is normal if and only if $\left(H_{0}, \mathfrak{h}_{1}\right)$ does not belong to Table 1

Corollary 4.7. Under the hypothesis of the previous theorem and if $\mathfrak{h}$ is of classical type, then $\mu^{-1}(0)$ is normal

From [Pan, Theorem 3.2] (or, alternatively, Remark 1.8 and Corollary 1.7), (i) holds. The remaining of the section is devoted to the proof of Theorem 4.6 (ii). 


\begin{tabular}{|c|c|c|c|c|c|c|}
\hline Type & \multicolumn{3}{|c|}{ Kac diagram } & $\mathrm{m}$ & $n$ & $\mathfrak{w}$ \\
\hline$E_{6}^{(1)}$ & \multicolumn{3}{|c|}{$\begin{array}{ll}1 & 1\end{array}$} & 9 & $\begin{array}{l}x_{\alpha_{2}+\alpha_{4}} \\
+x_{\alpha_{3}+\alpha_{4}} \\
+x_{\alpha_{5}+\alpha_{4}}\end{array}$ & $\left\langle x_{\alpha_{4}}\right\rangle$ \\
\hline$E_{7}^{(1)}$ & & 1 & 1 & 14 & idem & idem \\
\hline \multirow{3}{*}{$E_{8}^{(1)}$} & & $\begin{array}{ll}1 & 0 \\
& 1\end{array}$ & & 24 & idem & idem \\
\hline & 1 & $\begin{array}{ll}1 & 0 \\
& 1\end{array}$ & & 20 & $\begin{array}{l}x_{\alpha_{4}+\alpha_{5}+\alpha_{6}} \\
+x_{\alpha_{6}+\alpha_{7}} \\
\end{array}$ & $\left\langle x_{\alpha_{6}}\right\rangle$ \\
\hline & & 0 & & 15 & $\begin{array}{l}x_{\alpha_{2}+\alpha_{3}+\alpha_{4}} \\
+x_{\alpha_{2}+\alpha_{4}+\alpha_{5}} \\
+x_{\alpha_{5}+\alpha_{6}+\alpha_{7}} \\
+x_{\alpha_{7}+\alpha_{8}} \\
\end{array}$ & $\left\langle x_{\alpha_{2}}, x_{\alpha_{7}}\right\rangle$ \\
\hline
\end{tabular}

\begin{tabular}{|c|c|}
\hline Type & Kac diagram \\
\hline$G_{2}^{(1)}$ & $\begin{array}{l}\square \\
0\end{array}$ \\
\hline$F_{4}^{(1)}$ & $\begin{array}{l}\mathrm{O}-\mathrm{O}-\mathrm{O}-\mathrm{O}-\mathrm{O} \\
1 \\
1\end{array}$ \\
\hline$E_{6}^{(2)}$ & $\begin{array}{lllll}\mathrm{O}-\mathrm{O} & \mathrm{O} & \mathrm{O}-\mathrm{O} \\
1 & 1 & 0 & 1\end{array}$ \\
\hline
\end{tabular}

ceptional normal

TABLE 1. Exceptional non-normal

For this, we first rephrase condition (5) in Lemma 4.8. Then we list all the $\theta$ representations of rank one in Proposition 4.10. In Lemma 4.11, we check which of them satisfies condition $(\mathcal{P})$. Finally, we check that the non-normal cases of rank one do not yield any non-normal example of greater rank in Lemma 4.12.

Recall that all Cartan subspaces of $\mathfrak{h}_{1}$ are conjugate. From Lemma 4.5 (i) (iii) and Proposition 4.3 (iv), the decomposition class $J\left(\mathfrak{h}^{\mathfrak{c}},\{0\}\right)$ is the only one with maximal modality $\bmod \left(H_{0}, \mathfrak{h}_{1}\right)=\operatorname{dim} \mathfrak{c}$. Moreover, the decomposition classes with $\bmod \left(H_{0}, J(\mathfrak{l}, \mathcal{O})\right)=\operatorname{dim} \mathfrak{c}-1$ are the ones such that $\operatorname{dim}(\mathfrak{z} \mathfrak{l})_{1}=\operatorname{dim} \mathfrak{c}-1$ and $\mathcal{O}$ arbitrary. The condition on $\mathfrak{l}$ means that $\left(L_{0}, \mathfrak{l}_{1}^{\prime}\right)$ is a $\theta$-representation of rank one, where $\mathfrak{l}^{\prime}$ is the derived subalgebra of $\mathfrak{l}$.

Recall that, for $x \in \mathfrak{h}_{1}$ with $\mathfrak{l}=\mathfrak{h}^{x_{s}}$, we have $\mathfrak{h}_{0}^{x, y} \subset \mathfrak{h}^{x} \subset \mathfrak{l}$ and that $\mathfrak{l}_{0}=\mathfrak{l}_{0}^{\prime}$ by Lemma 4.5 (iv). Hence, using Corollary 1.7 (v), we have:

Lemma 4.8. Under hypothesis of Theorem 4.6, $\mu^{-1}(0)$ is normal if and only if

$$
\forall n \in \mathfrak{l}_{1}^{\prime} \text { nilpotent, } \exists n^{\prime} \in\left(\mathfrak{l}^{\prime}\right)_{-1}^{n} \text { such that }\left(\mathfrak{l}^{\prime}\right)_{0}^{\left(n, n^{\prime}\right)}=\{0\} .
$$

for any Levi subalgebra $\mathfrak{l} \subset \mathfrak{h}$ arising from $\mathfrak{h}_{1}$ such that $\left(L_{0}, \mathfrak{l}_{1}^{\prime}\right)$ is of rank one:

In other words, $\mu^{-1}(0)$ is normal if and only if the same holds for the semisimple part of any Levi subalgebra arising from $\mathfrak{h}_{1}$ whose associated $\theta$-representation is of rank one. The main difference with the symmetric Lie algebra case studied in [Pan] is that we have many $\theta$-representations of rank one. We now aim to classify them. For this, we recall a few features of the so-called Kac diagrams. The reader is referred to [Kac, §3] or [Vin, §8] for a detailed treatment.

- A Kac diagram is an affine (possibly twisted) Dynkin diagram $X_{n}^{(\ell)}$ equipped with an integer label for each node. Up to isomorphisms of representations, it is enough to consider labels in $\{0,1,2\}$. If we focus on representations of positive rank, one can restricts to labels in $\{0,1\}$.

- If $X_{n}(\ell)$ with a labelling $\left(v_{0}, \ldots, v_{n}\right)$ form a Kac diagram, then it gives rise to a $\mathbb{Z}_{m}$-grading on a reductive Lie algebra $\mathfrak{h}$, where $m=\ell \sum v_{i}$. 
- The Lie algebra $\mathfrak{h}_{0}$ is a reductive algebra whose semisimple part is the Lie algebra corresponding to the subdiagram of 0-labelled nodes. The rank of $\mathfrak{h}_{0}$ is that of the finite Dynkin diagram giving rise to $X_{n}^{(\ell)}$.

For instance, for each affine Dynkin diagram $X_{n}^{(\ell)}$ appearing in the classification of Kac [Kac, §3, Table 1], we can label each node of $X_{n}^{(\ell)}$ with 1 . We denote such a Kac diagram by $X_{n}^{(\ell)}(\underline{1})$. They give rise to the only $\theta$-representations of positive rank $\left(H_{0}, \mathfrak{h}_{1}\right)$ where $H_{0}$ is a torus.

Lemma 4.9. Let $\left(H_{0}, \mathfrak{h}_{1}\right)$ be a $\theta$-representation corresponding to a Kac diagram of the form $X_{n}^{(\ell)}(\underline{1})$. Then $\left(H_{0}, \mathfrak{h}_{1}\right)$ is stable locally free of rank 1 , and $\mu^{-1}(0)$ is a normal variety.

Proof. In this case, $\mathfrak{h}_{0}$ is just the Cartan subalgebra of $\mathfrak{h}$ (associated with the Dynkin diagram without the extended node $\alpha_{n+1}$ ) and $\mathfrak{h}_{1}$ is the sum of root spaces $\mathfrak{h}_{\alpha_{i}}, i \in \llbracket 1, n+1 \rrbracket$. Local freeness is clear since $\alpha_{1}, \ldots \alpha_{n}$ is a basis of $\mathfrak{h}_{0}^{*}$. By Lemma 4.5 (ii), the rank of $\left(H_{0}, \mathfrak{h}_{1}\right)$ is one. Since $\alpha_{n+1}$ is the opposite of the highest root, there is a single relation linking the $\alpha_{i}: \alpha_{n+1}=\sum_{i \in \llbracket 1, n \rrbracket} a_{i} \alpha_{i}$ with $a_{i}<0$. By Corollary $3.3,\left(H_{0}, \mathfrak{h}_{1}\right)$ is stable and $\mu^{-1}(0)$ is normal.

Proposition 4.10. Let $\left(H_{0}, \mathfrak{h}_{1}\right)$ be a stable and locally free $\theta$-representation of rank 1 obtained from a simple Lie algebra $\mathfrak{h}$. Then $\left(H_{0}, \mathfrak{h}_{1}\right)$ corresponds to a Kac diagram of the form $X_{n}^{(\ell)}(\underline{1})$ or which appears in Table 1 or Table 2

Proof. When the Lie algebra $\mathfrak{h}$ is exceptional we use the software [GAP] and its package [SLA]. We proceed as follows ${ }^{1}$.

Given $\mathfrak{h}$ of exceptional type (respectively of type $D_{4}$ ), the first step consists in listing the Kac-diagrams relative to $\mathfrak{h}$ (resp. with underlying Dynkin diagram $\left.D_{4}^{(3)}\right)$ with labels in $\{0,1\}$. Then we select the corresponding gradings for which $\operatorname{dim} \mathfrak{h}_{1}=\operatorname{dim} \mathfrak{h}_{0}+1$. There are few of them. For each selected grading, we choose a random element $x \in \mathfrak{h}_{1}$ and compute its semisimple part $s$. Since the rank of $\left(H_{0}, \mathfrak{h}_{1}\right)$ is at least 1 , the element $s$ should be non-zero (otherwise choose another $x$ ). Then, $\left(H_{0}, \mathfrak{h}_{1}\right)$ is stable locally free of rank 1 if and only if $\operatorname{dim} \mathfrak{h}_{1}^{s}=1$ (Lemma 4.5). In addition to the $X_{n}^{(\ell)}(\underline{1})$, the only other Kac diagrams arising in this way are those of Tables 1 and 2 .

Assume now that $\mathfrak{h} \subset \mathfrak{g l}(V)$ is classical. Our goal is to show that the only stable and locally free $\theta$-representatons of rank 1 are those arising from the $X_{n}^{(\ell)}(\underline{1})$. The strategy is similar to the exceptional case, except that we rather rely on the description of classical $\theta$-representations of Vinberg [Vin, §7]. We refer to this paper and we use the notation therein. In particular, there are four subcases to consider: First case: $\theta$ is an inner automorphism of $\mathfrak{h}=\mathfrak{s l}(V)$ (type $A)$;

Second case: $\mathfrak{h}$ is of type $B$ or $D$;

Third case: $\mathfrak{h}$ is of type $C$;

Fourth case: $\theta$ outer and $\mathfrak{h}$ of type $A$.

\footnotetext{
${ }^{1}$ The detail of the GAP-computations used in Propositions 4.10 and Lemmas 4.11 and 4.12 can be found in the auxilary file "computations_rk1.txt"
} 
In the first three cases (respectively in the fourth case) $\theta$ (respectively $\theta^{2}$ ) is an automorphism of order $m_{0}$ given by $g \mapsto a g a^{-1}$ for some $a \in \mathrm{GL}(\mathrm{V})$ satisfying $a^{m_{0}} \in\{ \pm I d\}$. For $\theta$ of order 1 or 2 , the only locally free case of rank 1 is $A_{1}^{(1)}(\underline{1})$ so we may assume that $m_{0}>1$. Then $V=\bigoplus_{\lambda \in S} V(\lambda)$ where $V(\lambda)=\{x \in V \mid a . x=$ $\lambda x\}$ and $S=\left\{\lambda \mid \lambda^{m_{0}}=+1\right\}$ or $S=\left\{\lambda \mid \lambda^{m_{0}}=-1\right\}$. That is $S=\left\{\lambda_{0} \omega_{0}^{j} \mid j \in \mathbb{Z}_{m_{0}}\right\}$ for $\lambda_{0}=1$ or $\exp \left(\frac{2 i \pi}{2 m_{0}}\right)$ and $\omega_{0}:=\exp \left(\frac{2 i \pi}{m_{0}}\right)$. For $j \in \mathbb{Z}_{m_{0}}$, we set $\lambda_{j}:=\lambda_{0} \omega_{0}^{j}$ and $k_{j}:=\operatorname{dim} V\left(\lambda_{j}\right)$. The elements $x \in \mathfrak{h}_{k}$ satisfy $x . V\left(\lambda_{j}\right) \subset V\left(\lambda_{j+k}\right)$ for any $j \in \mathbb{Z}_{m_{0}}$.

We first look at the first case. From [Vin], the rank of $\left(H_{0}, \mathfrak{h}_{1}\right)$ is $\min _{j \in \mathbb{Z}_{m_{0}}} k_{j}$. Since $\left.\mathfrak{h}_{0} \cong \mathfrak{s l}(V) \cap \prod_{j \in \mathbb{Z}_{m_{0}}} \mathfrak{g l}\left(V_{j}\right)\right)$ and $\mathfrak{h}_{1} \cong \prod_{j \in \mathbb{Z}_{m_{0}}} \operatorname{Hom}\left(V_{j}, V_{j+1}\right)$, we have

$\operatorname{dim} \mathfrak{h}_{1}-\operatorname{dim} \mathfrak{h}_{0}=\left(\sum_{j \in \mathbb{Z}_{m_{0}}} k_{j} k_{j+1}\right)-\left(\sum_{j \in \mathbb{Z}_{m_{0}}} k_{j}^{2}-1\right)=1-\frac{1}{2} \sum_{j \in \mathbb{Z}_{m_{0}}}\left(k_{j}-k_{j+1}\right)^{2}$.

From Lemma 4.5 (ii), we want that this number is 1 . The only possibility is that $k_{j}=k_{j^{\prime}}$ for any $j, j^{\prime} \in \mathbb{Z}_{m_{0}}$ and the condition on the rank forces this common value to be 1 . In this particular case, $H_{0}$ is a torus so this must coincide with a grading given by a Kac diagram of the form $A_{n}^{(1)}(\underline{1})$ (inner automorphism).

From now on we focus on the last three cases. In each of these cases, a nondegenerate bilinear form comes into play. This form induces a duality between $V(\lambda)$ and $V(\bar{\lambda})$ and we have $k_{j}=k_{\bar{j}}$ where $\bar{j}$ denotes the element of $\mathbb{Z}_{m_{0}}$ satisfying $\lambda_{\bar{j}}=\overline{\lambda_{j}}$. Depending on whether each elements 1 and -1 belong to $S$ or not, the corresponding $\theta$-representations may have different behavior. For $\nu \in\{ \pm 1\}$, we set $\eta_{\nu}:=\left\{\begin{array}{ll}1 & \text { if } \nu \in S \\ -1 & \text { if } \nu \notin S\end{array}\right.$. Set also $\epsilon_{1}:=\left\{\begin{array}{ll}1 & \text { in the } 2 \text { nd and } 4 \text { th case } \\ -1 & \text { in the } 3 \text { rd case }\end{array}\right.$ and $\epsilon_{-1}:=\left\{\begin{array}{ll}1 & \text { in the } 2 \text { nd case } \\ -1 & \text { in the } 3 \text { rd and } 4 \text { th case }\end{array}\right.$. We then have a uniform formula

$$
\begin{aligned}
\operatorname{dim} \mathfrak{h}_{1}-\operatorname{dim} \mathfrak{h}_{0} & =\frac{1}{2}\left[\sum_{j} k_{j} k_{j+1}-\sum_{j} k_{j}^{2}+\left(\epsilon_{1} \eta_{1} k_{0}+\epsilon_{-1} \eta_{-1} k_{m^{\prime}}\right)\right] \\
& =\frac{1}{4}\left[-\sum_{j}\left(k_{j}-k_{j+1}\right)^{2}+2 \epsilon_{1} \eta_{1} k_{0}+2 \epsilon_{-1} \eta_{-1} k_{m^{\prime}}\right]
\end{aligned}
$$

In a nutshell, $\mathfrak{h}_{0}$ is a product of the $\mathfrak{g l}\left(V_{j}\right) \cong \mathfrak{g l}\left(V_{\bar{j}}\right)$ for $\lambda_{j} \notin\{ \pm 1\}$ and possible copies of $\mathfrak{s o}\left(V_{j}\right)$ or $\mathfrak{s p}\left(V_{j}\right)$ for $\lambda_{j} \in\{ \pm 1\}$. A similar combinatoric holds for $\mathfrak{h}_{1}$. In the fourth case, the assumption $m_{0}>1$ ensures that $\mathbb{k} I d_{V}$ lies in a $k$-th part of the grading in $\mathfrak{g l}(V)$ satisfying $k \notin\{0,1\}$.

We choose $m^{\prime} \in \mathbb{Z}_{m_{0}}$ such that $\operatorname{Re}\left(\lambda_{m^{\prime}}\right)$ is minimal. By convention, the argument of a complex number is taken in $\left[0,2 \pi\left[\right.\right.$. Choosing $j_{\text {min }} \in \mathbb{Z}_{m_{0}}$ such that $k_{j}$ is minimal and $\operatorname{Im}\left(\lambda_{j}\right) \geqslant 0$, we define

$$
\begin{aligned}
& I=\left\{j \mid \arg \left(\lambda_{j}\right)<\arg \left(\lambda_{j_{\text {min }}}\right) \text { or } \arg \left(\lambda_{m^{\prime}}\right) \leqslant \arg \left(\lambda_{j}\right)<\arg \left(\lambda_{\overline{j_{m i n}}}\right)\right\}, \\
& I=\left\{j \mid \arg \left(\lambda_{j_{m i n}}\right) \leqslant \arg \left(\lambda_{j}\right)<\arg \left(\lambda_{m^{\prime}}\right) \text { or } \arg \left(\lambda_{\overline{j_{m i n}}}\right) \leqslant \arg \left(\lambda_{j}\right)\right\} .
\end{aligned}
$$


Using the equality $n^{2}=n(n-1)+n$, we can write

$$
\begin{aligned}
\operatorname{dim} \mathfrak{h}_{1}-\operatorname{dim} \mathfrak{h}_{0}=\frac{1}{4} & {\left[-\sum_{j \in I}\left(k_{j}-k_{j+1}\right)\left(k_{j}-k_{j+1}-1\right)-\sum_{j \in J}\left(k_{j+1}-k_{j}\right)\left(k_{j+1}-k_{j}-1\right)\right.} \\
& \left.+\sum_{j \in I}\left(k_{j+1}-k_{j}\right)+\sum_{j \in J}\left(k_{j}-k_{j+1}\right)+2 \epsilon_{1} \eta_{1} k_{0}+2 \epsilon_{-1} \eta_{-1} k_{m^{\prime}}\right]
\end{aligned}
$$

The first two terms are non-positive and the rest of the expression is equal to $\left(k_{j_{m i n}}-k_{0}\right)+\left(k_{\overline{j_{m i n}}}-k_{m^{\prime}}\right)+\left(k_{j_{\text {min }}}-k_{m^{\prime}}\right)+\left(k_{\overline{j_{m i n}}}-k_{0}\right)+2 \epsilon_{1} \eta_{1} k_{0}+2 \epsilon_{-1} \eta_{-1} k_{m^{\prime}}=$ $2\left[\left(\epsilon_{1} \eta_{1}-1\right) k_{0}+k_{j_{m i n}}\right]+2\left[\left(\epsilon_{-1} \eta_{-1}-1\right) k_{m^{\prime}}+k_{j_{m i n}}\right]$. Each of the terms in brackets is either equal to $k_{j_{\text {min }}}$ if $\epsilon_{\nu} \eta_{\nu}=1$, or is not greater than $-k_{j_{\text {min }}}$ if $\epsilon_{\nu} \eta_{\nu}=-1$. In particular, $\operatorname{dim} \mathfrak{h}_{1}-\operatorname{dim} \mathfrak{h}_{0}$ can be positive only if $\epsilon_{\nu} \eta_{\nu}=1$ for $\nu \in\{ \pm 1\}$. Hence, it is of type I in the terminology of [Vin, §7.2], and its rank is $k_{j_{m i n}}$. Then, it follows from our formulas and Lemma 4.5 that the only locally free cases of rank 1 are the following ones

$$
\left(\begin{array}{c}
k_{j_{\text {min }}}=1, \\
\forall j \in I, k_{j}-k_{j+1} \in\{0,1\} \\
\forall j \in J, k_{j+1}-k_{j} \in\{0,1\}
\end{array}\right) \text { and }\left\{\begin{array}{ll} 
\pm 1 \in S & \text { in the } 2 \text { nd case } \\
\pm 1 \notin S & \text { in the 3rd case } \\
1 \in S,-1 \notin S & \text { in the 4th case }
\end{array} .\right.
$$

In the last three cases, the action is not stable in general. Given subspaces $U(\lambda) \subset V(\lambda)$ of dimension 1, we can construct a non-zero semisimple element $C \in \mathfrak{h}_{1}$ such that $C(U(\lambda))=U\left(\omega_{0} \lambda\right)$. Then $V^{\prime}:=\operatorname{Ker}(C)$ decomposes as $V^{\prime}:=\bigoplus_{\lambda} V^{\prime}(\lambda)$; the decomposition in two summands $V=\left(\bigoplus_{\lambda} U(\lambda)\right) \oplus V^{\prime}$ is orthogonal and the derived subalgebra of $\mathfrak{g l}(V)^{C}$ can be identified with $\mathfrak{s l}\left(V^{\prime}\right)$. If there exists $j \in \mathbb{Z}_{m_{0}}$ such that $k_{j}, k_{j+1} \geqslant 2$, then we can construct in each case a non-zero element $N$ in $\mathfrak{h}_{1}^{C} \cap \mathfrak{s l}\left(V^{\prime}\right)$ with $N\left(V^{\prime}\left(\lambda_{j}\right)\right) \subset V^{\prime}\left(\lambda_{j+1}\right), N\left(V^{\prime}\left(\lambda_{\overline{j+1}}\right)\right) \subset V^{\prime}\left(\lambda_{\bar{j}}\right)$ and $N(U(\lambda))=0=N\left(V^{\prime}\left(\lambda_{k}\right)\right)$ for any $\lambda \in S$ and any $k \notin\{j, \overline{j+1}\}$. This contradicts the stability hypothesis since $N \in \mathfrak{h}^{C} \backslash \mathbb{k} C$, see Lemma 4.5 (iii). Thus, the only stable gradings to consider are those with $\begin{cases}k_{j}=1 & \text { for } j \text { such that } \lambda_{j} \notin\{ \pm 1\} \\ k_{j} \in\{1,2\} & \text { for } j \text { such that } \lambda_{j} \in\{ \pm 1\}\end{cases}$ The different possibilities are listed in Table 3 (up to multiplication of $a$ by -1 in type $B$, which gives rise to the same grading). Once again, $H_{0}$ is a torus in each case so the corresponding grading must be obtained from a Kac diagram of the form $X_{n}^{(\ell)}(\underline{1})$. These Kac diagrams are identified in the last column of Table 3.

Lemma 4.11. If $\left(H_{0}, \mathfrak{h}_{1}\right)$ is a $\theta$-representation associated to a Kac diagram of Table 1 (resp. Table 2) then it does not satisfy (resp. it satisfies) condition ( $\mathcal{P}$ ) of Lemma 4.8 .

The proof of this lemma relies on computer-based computations. First, we list representatives of the nilpotent $H_{0}$-orbits on $\mathfrak{h}_{1}$. For any such representative $n_{i}$, we choose a random element $n_{i}^{\prime} \in \mathfrak{h}_{-1}^{n}$. Then we compute $\mathfrak{h}_{0}^{\left(n_{i}, n_{i}^{\prime}\right)}$. It turns out to be $\{0\}$ for each $i$ in cases of Table 2 so $(\mathcal{P})$ holds. In cases of Table 1 , we compute

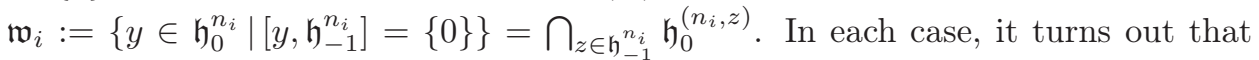
there exists $i$ such that $\mathfrak{w}_{i} \neq\{0\}$. Hence $(\mathcal{P})$ must fail in these cases. An example 


\begin{tabular}{|c|c|c|c|c|}
\hline Case & $\begin{array}{l}\text { Parity } \\
\text { of } m_{0}\end{array}$ & $\begin{array}{l}\text { Parity of } \\
\operatorname{dim}(V)\end{array}$ & Condition on the $k_{j}$ & $X_{n}^{(\ell)}(\underline{1})$ \\
\hline First case & 1 & 1 & $\forall j, k_{j}=1$ & $A_{n}^{(1)}(\underline{1})$ \\
\hline \multirow{3}{*}{ Second case } & \multirow{3}{*}{ even } & odd & $k_{j}= \begin{cases}2 & \text { if } j=0 \\
1 & \text { else }\end{cases}$ & $B_{n}^{(1)}(\underline{1})$ \\
\hline & & \multirow{2}{*}{ even } & $\forall j, k_{j}=1$ & $D_{n}^{(1)}(\underline{1})$ \\
\hline & & & $k_{j}= \begin{cases}2 & \text { if } j \in\left\{0, \frac{m_{0}}{2}\right\} \\
1 & \text { else }\end{cases}$ & $D_{n}^{(2)}(\underline{1})$ \\
\hline Third case & even & even & $\forall j, k_{j}=1$ & $C_{n}^{(1)}(\underline{1})$ \\
\hline \multirow[t]{2}{*}{ Fourth case } & \multirow[t]{2}{*}{ odd } & even & $k_{j}= \begin{cases}2 & \text { if } j=0 \\
1 & \text { else }\end{cases}$ & $A_{2 p-1}^{(2)}(\underline{1})$ \\
\hline & & odd & $\forall j, k_{j}=1$ & $A_{2 p}^{(2)}(\underline{1})$ \\
\hline
\end{tabular}

TABLE 3 . Stable classical locally free grading of rank 1

of such $n_{i}, \mathfrak{w}_{i}$ is given in the table, using the numbering of the roots as in [Bo] and where $x_{\alpha}$ denotes a root vector for the root $\alpha$.

Lemma 4.12. Let $\left(H_{0}, \mathfrak{h}_{1}\right)$ be a locally free stable $\theta$-representation with $\mathfrak{h}$ simple. Then there is no proper Levi $\mathfrak{l}$ arising from $\mathfrak{h}_{1}$ such that $\left(L_{0}, \mathfrak{l}_{1}^{\prime}\right)$ corresponds to a Kac diagram of Table 1.

Proof. Assume that such a proper Levi exists. Then $\mathfrak{h}$ is of type $E_{7}$ or $E_{8}$ and the Cartan subspace of $\mathfrak{h}_{1}$ is of dimension 2 or 3 . Moreover the order of the automorphism $\theta$ giving rise to the graduation is a multiple of 9 or 14 . We can now consider all the possible Kac diagrams of type $E_{7}$ or $E_{8}$ such that $\operatorname{dim} \mathfrak{g}_{1}-\operatorname{dim} \mathfrak{g}_{0} \geq 2$. After computation, it turns out that the resulting $\mathbb{Z}_{m}$-gradings have order which are not multiple of 9 or 14 , which is a contradiction.

Remark 4.13. Conjecture 1.3 in the case of a locally free stable $\theta$-representation follows from [BLLT, Theorem 1.2]. We briefly explain here how to recover it using the results of this paper. First, note that the 5 exceptional cases of Table 1 are of rank one. Then it follows from [BLLT, Proposition $3.4 \& 5.1$ ] that the natural map $\mathfrak{c} \oplus \mathfrak{c}^{\vee} / W \rightarrow V \oplus V^{*} / / G$ is a dominant closed immersion, hence an isomorphism. In the other cases, Theorem 4.6 ensures that $\mu^{-1}(0)$ is normal. So the same holds for the symplectic reduction and the result follows from [BLLT, Proposition 5.1] and Remark 1.4.

Note that the ground results on polar representations of [DK] are known for $\theta$ representations over any algebraically closed field of characteristic zero. Thus, we don't need the assumption $\mathbb{k}=\mathbb{C}$ used in [BLLT].

\section{REFERENCES}

[Bo] N. Bourbaki, Groupes et algèbres de Lie, Chapitres 4, 5 et 6 Masson, 1981.

[Br] A. Broer, Lectures on decomposition classes. In: Representation theories and algebraic geometry (Montreal, PQ, 1997), NATO Adv. Sci. Inst. Ser. C Math. Phys. Sci., 514, Kluwer Acad. Publ., 1998, 39-83. 
[Bu11] M. Bulois. Irregular locus of the commuting variety of reductive symmetric Lie algebras and rigid pairs. Transform. Groups, 16:no. 4 (2011), 1027-106

[BLLT] M. Bulois, C. Lehn, M. Lehn, R. Terpereau. Towards a symplectic version of the Chevalley restriction theorem. preprint, arXiv:1604.04121.

[DK] J. Dadok and V. Kac. Polar representations. Journal of Algebra, 92:504-524, 1985.

[GAP] The GAP Group, GAP - Groups, Algorithms, and Programming, Version 4.8.3; 2016, (http: 八protect \kern-.1667em\relax/www.gap-system.org)

[HSS] H.-C. Herbig, G. W. Schwartz and C. Seaton. Symplectic quotients have symplectic singularities. arXiv: 170602089, 2017.

[Jos] A. Joseph. On a Harish-Chandra homomorphism. Comptes Rendus de l'Académie des Sciences - Series I - Mathematics, 324, Issue 7:759-764, 1997.

[Kac] V. Kac. Some remarks on nilpotent orbits. J. Algebra, 64, no.1:190-213, 1980.

[Pan] D. Panyushev. The Jacobian modules of a representation of a Lie algebra and geometry of commuting varieties. Compos. Math., 94, Issue 2:181-199, 1994.

[Po] V. L. Popov, Irregular and singular loci of commuting varieties, Transform. Groups, 13 (2008), 819-837.

[SB] G. W. Schwarz, and M. Brion. Théorie des invariants et géométrie des variétés quotients. Travaux en cours, vol. 61. Hermann, Paris, 2000.

[SLA] W. de Graaf, SLA - a GAP package, Version 1.2 , 2016, http: \protect\kern-.1667em\relax/www.science.unitn.it/ degraaf/sla.html

[Vin] E. Vinberg. The Weyl group of a graded Lie algebra. Math. USSR Izvestija, 10:463-495, No.3, 1976.

[TY] P. Tauvel and R. W. T. Yu. Lie algebras and algebraic groups, Springer Monographs in Mathematics, Springer-Verlag, 2005.

Michael Bulois, Univ Lyon, UjM-Saint-Etienne, CNRS UMR 5208, Institut Camille Jordan, 10 rue Tréfilerie, CS 82301, F-42023 Saint-Etienne Cedex 2, France

E-mail address: michael.bulois@univ-st-etienne.fr 